\title{
SOME RECENT DISCOVERIES IN THE ISOMORPHIC THEORY OF BANACH SPACES ${ }^{1}$
}

\author{
BY HASKELL P. ROSENTHAL
}

\section{Contents}

1. Informal discussion of the fundamental results Characterizations of Banach spaces containing $l^{1}$

2. Sequences of functions with no point-wise convergent subsequence

3. Point-wise compact subsets of the first Baire class

Proof of the fundamental theorem

References

1. We shall focus on recent progress concerning one fundamental problem in Banach space theory. In so doing, we will be neglecting a vast amount of remarkable current research. In particular, we will be neglecting strong recent work on the structure of uniformly convex spaces, the location of nice finite-dimensional subspaces in general spaces, and injectivity. Before passing to our main considerations, we would just like to indicate the nature of the advances in these three other directions.

The work on uniformly convex Banach spaces is due to G. Pisier [46] based on earlier work of R. C. James [30] and P. Enflo [15]. It makes essential use of martingale theory to get powerful norm estimates on general uniformly convex spaces. Pisier showed that every uniformly convex space admits an equivalent norm such that all its two-dimensional spaces are "power-type" smooth and convex. Enflo had previously obtained the uniform smoothness and convexity without the power-type estimates, while James showed that nonreflexive Banach spaces always have two-dimensional spaces whose unit balls are almost square, i.e. as far from being uniformly convex or smooth as is possible. Precisely, we have the

THEOREM. If a Banach space $B$ is uniformly convex, then it admits an equivalent norm $\|\cdot\|$ so that there are $\delta, p$ and $q$ with $\delta>0$ and $1<q \leqslant 2<$ $p<\infty$ such that for all $x$ and $y$ in $B$,

$$
\|x\|^{p}+\delta\|y\|^{p} \leqslant \frac{\|x+y\|^{p}+\|x-y\|^{p}}{2}
$$

This is an expanded version of an invited address presented at the annual meeting of the American Mathematical Society held at Washington, D.C., on January 23, 1975; received by the editors November 10, 1977. Many of the results discussed were discovered after this meeting. In particular, much of the discussion in $\$ 3$ is concerned with the solution to a problem raised at the Washington lecture.

AMS (MOS) subject classifications (1970). Primary 43A15, 46C05, 46E15, 46E30, 46G10.

${ }^{1}$ Much of the work presented was partially supported by the National Science Foundation NSF-GP MCS 76-07459. 
and

$$
\|x\|^{q}+\delta^{-1}\|y\|^{q} \geqslant \frac{\|x+y\|^{q}+\|x-y\|^{q}}{2} .
$$

On the other hand, if $B$ has no equivalent uniformly convex norm, then for any equivalent norm $\|\cdot\|$ on $B$ and $\delta$ with $0<\delta<1$, there exist norm one elements $x$ and $y$ satisfying

$$
1+\delta=\|x\|+\delta\|y\| \leqslant \frac{\|x+y\|+\|x-y\|}{2} .
$$

The work on nicely placed finite-dimensional subspaces is due to J. L. Krivine [35] (see also [55] for an alternate exposition). This work provides a kind of substitute for the conjecture that every infinite dimensional Banach space $B$ contains a subspace isomorphic to $c_{0}$ or $l^{p}$ for some $1 \leqslant p<\infty$. (For counter-examples to the conjecture, see [61] and [18].) It appears to be an outgrowth of Krivine's earlier joint work with D. Dacunha-Castelle [10] on whether the conjecture is valid for $B$ a subspace of $L^{1}$, a problem which remains unsolved. ( $L^{1}$ denotes $L^{1}([0,1])$, the space of equivalence classes of Lebesgue integrable functions on the unit interval; $c_{0}$ the space of sequences of scalars vanishing at infinity; $l^{p}$ the space of sequences $\left(x_{j}\right)$ with $\sum_{j=1}^{\infty}\left|x_{j}\right|^{p}$ $<\infty$; "isomorphic" means "linearly homeomorphic".) Krivine's proof uses intuitions from probability theory. The techniques are analytical and combinatorial, not geometrical, and yield a new proof of the famous theorem of Dvoretzky that $l^{2}$ is finitely represented in every Banach space [13]. (Tzafriri [62] gave an earlier nongeometrical proof that some isomorph of $l^{2}$ is finitely represented in every Banach space.) Let $1 \leqslant p<\infty$ and $\left(x_{j}\right)$ a sequence of elements in some Banach space. Say that $l^{p}$ or $c_{0}$ is block finitely represented in $\left(x_{j}\right)$ if there exist arbitrarily long finite strings of successive linear combinations of the $x_{i}$ 's whose linear combinations are arbitrarily close in norm to the $l^{p}$ or $c_{0}$ norm. Precisely, if for every $\varepsilon>0$ there are $n$ finite subsets $F_{1}, \ldots, F_{n}$ of the positive integers $N$ with $\max F_{i}<\min F_{i+1}$ for all $1 \leqslant i \leqslant n-1$ and elements $b_{1}, \ldots, b_{n}$ with $b_{i}$ in the linear span of $\left\{x_{j}\right.$ : $\left.j \in F_{i}\right\}$ for all $i$ so that for all scalars $c_{1}, \ldots, c_{n}$,

$$
(1-\varepsilon)\left(\sum\left|c_{i}\right|^{p}\right)^{1 / p} \leqslant\left\|\sum c_{i} b_{i}\right\| \leqslant(1+\varepsilon)\left(\sum\left|c_{i}\right|^{p}\right)^{1 / p}
$$

(where $\left(\Sigma\left|c_{i}\right|^{p}\right)^{1 / p}=\sup \left|c_{i}\right|$ in the $c_{0}$ or " $p=\infty$ " case). Now Krivine's theorem may be stated as follows: Let $\left(x_{j}\right)$ be a sequence in a Banach space with infinite-dimensional linear span. Then either $l^{p}$ is block finitely represented in $\left(x_{j}\right)$ for some $1 \leqslant p<\infty$ or $c_{0}$ is block finitely represented in some permutation of $\left(x_{j}\right)$.

The discovery concerning injectivity is due to M. Zippin [64]. A Banach space $B$ is said to be injective if the space of scalars can be replaced by $B$ in the qualitative version of the Hahn Banach theorem; precisely, if for every Banach space $Z$, closed linear subspace $Y$, and bounded linear operator $T$ : $Y \rightarrow B$, there exists a bounded linear operator $\tilde{T}: Z \rightarrow B$ extending $T . B$ is said to be separably injective if $B$ is infinite-dimensional separable and the above extension property holds for all separable spaces $Z$. An early result of Sobczyk [57] established that $c_{0}$ is separably injective (for a short proof, see 
[63]). It's trivial that then any space isomorphic to $c_{0}$ is separably injective. Zippin has proved the converse: Every separable injective Banach space is isomorphic to $c_{0}$. The proof makes essential use of several discoveries in Banach space theory over the last 15 years. In particular, work of Amir [2], Pekczyński [44], Szlenk [60], Rosenthal [50] (see also [53]), Lindenstrauss (unpublished), Johnson and Zippin [33], and Alspach [1] is basic to the demonstration. Zippin's essential new ingredient is a remarkable approximation lemma concerning arbitrary Banach spaces $B$ with separable dual. The lemma shows that such a space $B$ may be embedded in $C([0,1])$ in such a way that its elements may be closely approximated by functions in a subspace isometric to $C(K)$ for some compact countable $K \subset[0,1],(C(K)$ denotes the space of continuous scalar valued functions on $K$ under the supremum norm). The techniques of his proof are functional-analytic and topological (in the point-set sense). For simplifications and some extensions, see [5]. No infinite-dimensional separable space is injective; in fact every such space contains a subspace isomorphic to $l^{\infty}$ (the space of all bounded sequences of scalars) [48]. The problem of classifying injective spaces up to isomorphism remains open: see [38] and [49] for a discussion of the known results.*

For the remainder, we shall deal with the following fundamental question, hereafter referred to as the Problem:

Does every infinite dimensional Banach space $B$ contain a subspace which is isomorphic to $c_{0}$, isomorphic to $l^{1}$, or reflexive and infinite dimensional?

The main progress so far effectively eliminates the $l^{1}$-case. The two most powerful results are as follows:

THEOREM 1. Let $\left(b_{j}\right)$ be a bounded sequence in some Banach space. Then either $\left(b_{j}\right)$ has a weak Cauchy subsequence or $\left(b_{j}\right)$ has a subsequence $\left(b_{j}^{\prime}\right)$ so that there is $a \delta>0$ with $\left\|\sum_{j=1}^{n} c_{j} b_{j}^{\prime}\right\| \geqslant \delta \sum_{j=1}^{n}\left|c_{j}\right|$ for every $n$ and scalars $c_{1}, \ldots, c_{n}$.

THEOREM 2. Let $B$ be a separable Banach space which contains no subspace isomorphic to $l^{1}$. Then every bounded subset of $B^{* *}$ is weak*-sequentially dense in its weak* closure.

A sequence $\left(b_{j}\right)$ in a Banach space $B$ is said to be a weak-Cauchy sequence if the scalar sequence $\left(b^{*}\left(b_{j}\right)\right)$ converges for every $b^{*} \in B^{*}$, the dual of $B$. A sequence $\left(b_{j}^{\prime}\right)$ satisfies the second alternative of Theorem 1 if and only if it is equivalent to the usual $l^{1}$ basis, that is, if for any sequence of scalars $\left(c_{j}\right)$, $\Sigma c_{j} b_{j}^{\prime}$ converges if and only if $\Sigma\left|c_{j}\right|<\infty$. Since the usual $l^{1}$-basis is not a weak-Cauchy sequence, the two alternatives of Theorem 1 are mutually exclusive.

Theorem 1 was proved by the author for the case of real scalars in [51]. Later, L. Dor obtained a proof for the complex scalars in [11], which also streamlined some of the arguments in [51]. We present a self-contained and hopefully motivated proof for Theorem 1 in \$2; the argument is real-variable and combinatorial, requiring only elementary ideas. Theorem 2 is a

*ADDED IN PROOF. For some recent fundamental progress, see R. Haydon, On dual $L^{1}$-spaces and injective bidual Banach spaces (to appear). 
consequence of Theorem 1 and work of J. Bourgain, D. H. Fremlin and M. Talagrand [7] based on earlier work of the author's concerning point-wise compact subsets of the first Baire class [54]. The techniques here are point-set topological and combinatorial. The base of the arguments is a beautiful characterization theorem published by $\mathrm{R}$. Baire in 1899 . We present the relevant ideas in \$3. (For a Banach space $X$, the weak*-topology on $X^{*}$ refers to the $X$-topology on $X^{*}$ in the natural pairing, while the weak-topology on $X$ refers to the $X^{*}$-topology on $X$. A subset $A$ of a topological space $Y$ is said to be sequentially dense in its closure if for every point $y$ in the closure of $A$ there exists a sequence $a_{1}, a_{2}, \ldots$ of elements of $A$ with $a_{n} \rightarrow y$ as $n \rightarrow \infty$.) For the remainder of the present section, we deduce some consequences of these two theorems, present other characterizations of Banach spaces containing $l^{1}$, and give some comments concerning the Problem. (For an application of Theorem 1 to Banach spaces and probability theory, see [8]. An extension of the result to "trees" of elements in Banach spaces is derived in [59].)

It is easily seen that if an infinite-dimensional $B$ has a separable dual $B^{*}$, then every bounded sequence in $B$ has a weak-Cauchy subsequence. A natural conjecture is that a separable $B$ contains a subspace isomorphic to $l^{1}$ if $B^{*}$ is nonseparable. This conjecture was disproved independently by $R$. C. James [31] and J. Lindenstrauss [37] (see also [22] for another counterexample). Nevertheless, there is a form of the conjecture, due to E. Odell and the author [42] which is valid: if $B^{*}$ is so nonseparable that the cardinality of $B^{* *}$ is larger than that of $B$, then $B$ does contain an isomorph of $l^{1}$. (The latter result follows easily from Theorem 2 as do most of the characterizations of spaces containing $l^{1}$ which we present.) An important open question related to the Problem is whether a separable $B$ contains a subspace isomorphic to $l^{1}$ if $Y^{*}$ is nonseparable for every infinite-dimensional subspace $Y$ of $B$. The following result of [32] may be of some use in isolating out the reflexive case in the Problem: If $B^{* *}$ is separable and $B$ is infinite dimensional, then $B$ contains an infinite-dimensional reflexive subspace.

It is a result of $R$. C. James [29] that the answer to the Problem is affirmative if $B$ has an unconditional basis. It is in fact an open question (which the author believes to be more difficult than the Problem) if every Banach space has a subspace with an unconditional basis. Theorem 1 reduces the latter problem to the case of spaces containing a nontrivial weakly null sequence (i.e. a sequence converging weakly but not in norm, to zero). Indeed, if $B$ contains no isomorph of $l^{1}$, let $\left(b_{n}\right)$ be a bounded sequence in $B$ with $\left\|b_{n}-b_{m}\right\| \geqslant 1$ for $n \neq m$. If $\left(b_{n}^{\prime}\right)$ is a weak-Cauchy subsequence, $\left(b_{2 n}^{\prime}-b_{2 n+1}^{\prime}\right)$ is a nontrivial weakly null sequence. It is proved in [56] that a nontrivial weakly null sequence has a subsequence with certain unconditional properties. However joint work of B. Maurey and the author [39] exhibits a nontrivial weakly null sequence with no unconditional subsequence (the question of whether such sequences exist was raised some time ago by $C$. Bessaga and A. Pekczyński [6]).

We pass now to some evident consequences of the Theorems. It is an immediate consequence of Theorem 1 that a Banach space contains an isomorph of $l^{1}$ if and only if it contains a bounded sequence with no 
weak-Cauchy subsequence. (This characterization was obtained by the author for both real and complex Banach spaces in [51], prior to the work in [11].) We then easily obtain the following consequence:

Corollary 1. Let $B$ be a weakly sequentially complete Banach space. Then either $B$ is reflexive or $B$ contains a subspace isomorphic to $l^{1}$.

Proof. Suppose that $B$ contains no isomorph of $l^{1}$ and let $\left(b_{j}\right)$ be a sequence of elements in the unit ball of $B$; i.e. $\left\|b_{j}\right\| \leqslant 1$ for all $j$. Then $\left(b_{j}\right)$ has a weak-Cauchy subsequence $\left(b_{j}^{\prime}\right)$. Since $B$ is weakly sequentially complete, $\left(b_{j}^{\prime}\right)$ converges weakly to some element in $B$. Thus the unit ball of $B$ is weakly sequentially compact, so by a standard result, $B$ is reflexive.

Any closed linear subspace of a weakly sequentially complete Banach space is also weakly sequentially complete. Now $L^{1}$ is a weakly sequentially complete Banach space (a rather nontrivial classical result). Thus if $B$ is a closed linear subspace of $L^{1}, B$ satisfies the conclusion of the Corollary. This result is due to M. I. Kadec and A. Pekzynnski [34]. Their elegant proof uses very natural special properties of $L^{1}$, but gives no hint that the Corollary holds in its general setting.

For the next result, we recall that a Banach space $B$ is said to have the Dunford-Pettis property if for every pair of weakly null sequences $\left(b_{j}\right)$ and $\left(b_{j}^{*}\right)$ in $B$ and $B^{*}$ respectively, $b_{j}^{*}\left(b_{j}\right) \rightarrow 0$ as $j \rightarrow \infty$. It can be seen that if $B$ has the D-P property and $\left(b_{j}\right)$ and $\left(b_{j}^{*}\right)$ are sequences in $B$ and $B^{*}$ respectively, then $b_{j}^{*}\left(b_{j}\right) \rightarrow 0$ as $j \rightarrow \infty$ provided one of the two sequences is weakly null and the other is weak-Cauchy. Perhaps the most important spaces with the D-P property are $L^{1}$ and $C([0,1])(\mathrm{cf}$. [12]).

Corollary 2. Suppose there exists a subspace $Y$ of $B^{*}$ with the D-P property so that $Y$ contains a nontrivial weakly null sequence. Then $B$ contains $a$ subspace isomorphic to $l^{1}$.

Proof. Choose a weakly null sequence $\left(y_{j}\right)$ of norm-one elements of $Y$. Choose a sequence $\left(b_{j}\right)$ of norm-one elements of $B$ with $y_{j}\left(b_{j}\right)>\frac{1}{2}$ for all $j$. In view of Theorem 1 , we merely need show that $\left(b_{j}\right)$ has no weak-Cauchy subsequence. Suppose to the contrary that $\left(b_{n_{j}}\right)$ were a weak-Cauchy subsequence. Then defining $y_{n}^{*}(y)=y\left(b_{n}\right)$ for all $j$ and $y \in Y,\left(y_{n_{i}}^{*}\right)$ would be a weak-Cauchy sequence in $Y^{*}$. Then by our observations concerning the D-P property, $y_{n_{j}}^{*}\left(y_{n_{j}}\right) \rightarrow 0$ as $j \rightarrow \infty$, yet $y_{n_{j}}^{*}\left(y_{n_{n}}\right) \geq \frac{1}{2}$ for all $j$, a contradiction.

This Corollary is due to H. Fakhoury [16]. Since $L^{1}$ has the D-P property yet contains a nontrivial weakly null sequence, Corollary 2 implies the result of A. Pełczyński [43] and J. Hagler [20]: if $B^{*}$ contains an isomorph of $L^{1}, B$ contains an isomorph of $l^{1}$. Pelczyński showed in [43] that the converse of this statement is true, using the fact that every separable Banach space is a continuous linear image of $l^{1}$ together with some reasoning involving injectivity. These considerations incidentally suggest the following question: Is there a Banach space $Y$ so that $Y$

(i) has the D-P property

(ii) contains a nontrivial weakly null sequence

(iii) is weakly sequentially complete

(iv) contains no subspace isomorphic to $L^{1}$ ? 
We pass now to a summary of most of the known characterizations of Banach spaces containing $l^{1}$, with some comments concerning certain nonseparable generalizations. ( $C$ denotes $C([0,1])$; for a set $\Gamma, l^{1}(\Gamma)$ (resp. $l^{\infty}(\Gamma)$ ) denotes the space of all scalar valued $f$ on $\Gamma$ with $\Sigma_{\gamma \in \Gamma}|f(\gamma)|<\infty$ (resp. $\left.\sup _{\gamma \in \Gamma}|f(\gamma)|<\infty\right)$.) lent:

THEOREM 3. Let $B$ be a separable Banach space. The following are equiva-

1. $B$ contains no subspace isomorphic to $l^{1}$.

2. $B$ is weak ${ }^{*}$-sequentially dense in $B^{* *}$.

3. The cardinality of $B^{* *}$ equals the cardinality of $B$.

4. Every bounded sequence in $B$ has a weak-Cauchy subsequence.

5. Every bounded sequence in $B^{* *}$ has a weak ${ }^{*}$-convergent subsequence.

6. Every bounded subset of $B$ is weakly sequentially dense in its weak closure.

7. Every bounded subset of $B^{* *}$ is weak ${ }^{*}$-sequentially dense in its weak* closure.

8. Every bounded weak*-closed convex subset of $B^{*}$ is the norm-closed convex hull of the set of its extreme points.

9. If $Y$ is a subspace of $B^{*}$ with the Dunford-Pettis property then every weakly-compact subset of $Y$ is norm-compact.

10. $B^{*}$ contains no subspace isomorphic to $L^{1}$.

11. $B^{*}$ contains no subspace isomorphic to $l^{1}(\Gamma)$ for any uncountable set $\Gamma$.

12. $C$ is not a continuous linear image of $B$.

Characterizations 4 and 5 are due to the author ([51] and [54]; see also [52] for a more direct proof of $1 \Rightarrow 5$ ); 2,3 and 8 are due jointly to E. Odell and the author [42], 6 and 7 follow from [54] and the work of J. Bourgain, D. H. Fremlin and M. Talagrand [7], 9 (which is equivalent to Corollary 2 above) is due to $H$. Fakhoury [16], and 10-12 are due to A. Pelczyński [43] and J. Hagler [20]. The characterizations 4, 8, 9 and 10 do not require the separability of $B$, nor does the following statement, also equivalent to 1 ([43] and [20]): $B^{*}$ contains no subspace isomorphic to $(C[0,1])^{*}$. The equivalence of 1 and 8 for general Banach spaces is due to R. Haydon [25]. Of course this equivalence is related to the result of Huff and Morris [28] based on a construction of Stegall [58]: For $B$ separable, $B^{*}$ is separable if and only if every norm-closed bounded convex subset of $B^{*}$ is the norm-closed convex hull of its extreme points. We do not know if $1 \Rightarrow 6$ for general Banach spaces. The remaining characterizations are all false for general Banach spaces, as may be seen by considering $B=c_{0}(\Gamma)$ for $\Gamma$ a set of cardinality the continuum, for $2,3,5,7$, and $11 .\left(c_{0}(\Gamma)\right.$ denotes the closure in $l^{\infty}(\Gamma)$ of all functions vanishing off a finite set.) Of course $1 \Rightarrow 12$ holds in general, but $12 \nRightarrow 1$ for e.g. $B=l^{\infty}$.

Here is a sketch of the proof of these equivalences modulo Theorem 2. It is easily seen that 7 implies all the remaining assertions with the exception of 8 . Indeed, $7 \Rightarrow 6$ is trivial, since the weak topology on $B$ is the same as the relative weak*-topology on $B$ regarded as a subset of $B^{* *}$. To see that $7 \Rightarrow 5$, let $\left(f_{n}\right)$ be a bounded sequence in $B^{* *}$.

By the weak*-compactness of bounded weak*-closed subsets of $B^{* *}$, there exists a point $f$ in $B^{* *}$ which is a weak*-cluster point of the sequence $\left(f_{n}\right)$. If 
infinitely many $f_{n}$ 's equal $f$, we are done. Otherwise, $f$ is in the weak ${ }^{*}$-closure of $A=\left\{f_{1}, f_{2}, \ldots\right\}$; hence there is a sequence of elements of $A$ which converges weak* to $f$; i.e. there are $n_{1}<n_{2}<\ldots$ with $f_{n_{i}} \rightarrow f$ weak*. $5 \Rightarrow 4$ follows trivially, by the same reasoning as $7 \Rightarrow 6.7 \Rightarrow 2$ in view of Goldstine's theorem. That is, if $A=\{b \in B:\|b\| \leqslant 1\}, A$ is weak*-dense in the unit ball of $B^{* *}$ (where $B$ is regarded as a subset of $B^{* *}$ ), so $A$ is sequentially dense in the unit ball of $B^{* *}$ which implies $2.2 \Rightarrow 3$ by a simple cardinality argument. Indeed, assuming $B$ is not the zero space, $B$ has cardinality $c$, the continuum. Now let $D$ be a countable norm-dense subset of $B^{*}$. Then it is easily seen that $D$ is also sequentially dense in $B^{* *}$, whence the cardinality of $B^{* *}$ is at most the cardinality of the set of all sequences of elements of $D$, which of course has cardinality $c .4 \Rightarrow 1$ since any sequence equivalent to the usual $l^{1}$-basis has no weak-Cauchy subsequence. $2 \Rightarrow 8$ follows from the Hahn-Banach theorem and results of Choquet on resultants [9] (see also $\$ 3$ and 12 of [45]). Suppose $A$ is a weak* compact convex subset of $B^{*}$; let $E$ be the set of extreme points of $A$ and suppose there is an element $a \in A$ which is not in the norm-closed convex hull of $E$. By the Hahn-Banach theorem there exists an $f \in B^{* *}$ with $f(a)>\sup _{e \in E} f(e)$. By the results of Choquet, there exists a probability measure $\mu$ on the Borel subsets of $A$ with $\mu(A \sim E)=0$ and $g(a)=\int g d \mu$ for each affine function $g$ on $A$ of the first Baire class. But $f$ is of the first Baire class on $A$ by 2 . Indeed, $f$ is the weak* limit of some sequence $\left(b_{n}\right)$ in $B$, and the $b_{n}$ 's may be regarded as continuous functions on A. Hence $f(a)=\int_{E} f d \mu \leqslant \sup _{e \in E} f(e)$, a contradiction. $4 \Rightarrow 9$ follows immediately from Corollary $2.9 \Rightarrow 10$ follows from the fact that $L^{1}$ has the D-P property (see e.g. [12]), while $10 \Rightarrow 12$ follows from the fact that $L^{1}$ is isometric to a subspace of $C^{*}$. Finally, to see that $7 \Rightarrow 11$, suppose there were an uncountable set $\Gamma$ with $l^{1}(\Gamma)$ isomorphic to a subspace of $B^{*}$. It follows by the Hahn-Banach theorem that there exists a weak* compact set $K \subset B^{* *}$ and a weak* continuous surjective map $\phi: K \rightarrow S$, where $S$ denotes the unit ball of $\left(l^{1}(\Gamma)\right)^{*}$ in its weak* topology. Now $S$ contains a set $A$ which is not weak*-sequentially dense in its closure. Indeed, $\left(l^{1}(\Gamma)\right)^{*}$ may be identified with $l^{\infty}(\Gamma)$. Letting $A$ denote the unit ball of $c_{0}(\Gamma)$, then $A$ is weak* dense in the unit ball of $l^{\infty}(\Gamma)$ but every element in the weak*-sequential closure of $A$ vanishes off some countable subset of $\Gamma$. It follows that $\phi^{-1}(A)$ is not sequentially dense in its weak*-closure, contradicting 7 . Thus 7 implies all the other assertions.

The fact that $1 \Rightarrow 7$ is the main point of Theorem 2, to be discussed in $\$ 3$. Assuming this fact, it is enough to show that all the assertions 2-12 are false if $B$ is separable and contains a subspace isomorphic to $l^{1}$. It is really quite simple to see this directly, except for the case of 12 and possibly 10 . It is enough to show that $3,6,8,10,11$ and 12 are false, from what has already been shown. Any separable Banach space is isometric to a quotient space $l^{1}$, hence so is $C$. It follows that $C^{*}$ is isometric to a subspace of $\left(l^{1}\right)^{*}=l^{\infty}$. Now $l^{1}([0,1])$ is isometric to a subspace of $C^{*}$, namely the space of all atomic finite signed Borel measures on $[0,1]$ (where $C^{*}$ is identified with the space of all finite signed Borel measures). Hence $l^{\infty}([0,1])$ is a continuous linear image of $\left(l^{1}\right)^{* *}$. Then $2^{c}=\operatorname{card} l^{\infty}([0,1]) \leqslant \operatorname{card}\left(l^{1}\right)^{* *} \leqslant \operatorname{card} B^{* *}$, establishing the failure of 3. Any infinite-dimensional Banach space $Y$ has the property 
that 0 is in the weak closure of $S=\{y \in Y:\|y\|=1\}$. Now if $Y$ is isomorphic to $l^{1}$, then any sequence in $Y$ which converges weakly to zero converges in norm (this result may be found in Banach's book [4]). Hence 0 is not in the weak-sequential closure of $S$, so 6 fails to hold. To see that 8 fails to hold, note that if a space $B$ satisfies 8 , then any Banach space isomorphic to a quotient of a subspace of $B$ also satisfies 8. But as observed already, assuming $l^{1}$ embeds in $B$, then $C$ is isomorphic to a quotient of a subspace of $B$. The atomic finite signed measures on $[0,1]$ of norm at most one constitute a norm-closed subset of $\left(C^{*}\right)$ which contains all the extreme points of the unit ball of $C^{*}$, hence $C$ fails 8 . Since the failure of 12 implies the failure of both 10 and 11 , we finally pass to $12 \Rightarrow 1$ (which is due to Pelczyński [43]). Let $X$ be an injective Banach space with $C \subset X$. (For example, $X=C^{* *}$ will do.) Supposing $B \supset Y$ isomorphic to $l^{\prime}$, let $T: Y \rightarrow C$ a bounded linear surjection. By injectivity there exists a bounded linear $\tilde{T}: B \rightarrow X$ extending $T$. Then $W=\tilde{T}(B)$ is separable and $W \supset C$. By a result of Pexzyński [44], there exists a subspace $Z$ of $C$ with $Z$ isomorphic to $C$ and a bounded linear projection $P$ from $W$ onto $Z$. The operator $P \tilde{T}$ is then a surjective map from $B$ onto an isomorph of $C$, establishing $12 \Rightarrow 1$.

We conclude this section with some remarks concerning possible nonseparable extensions. Since every sequence in $l^{1}$ which tends to zero weakly tends to zero in norm, if $l^{1}$ embeds in a Banach space $B$ then $B^{* *}$ contains a subspace in which every sequence tending to zero weak* tends to zero in norm. This motivates the following question:

Let $X$ be a Banach space so that $X^{*}$ has an infinite-dimensional closed linear subspace in which every sequence which tends to zero weak* tends to zero in norm. Does $X$ have a subspace isomorphic to $l^{1}(\Gamma)$ for some uncountable set $\Gamma$ ?

J. Hagler and W. B. Johnson show in [23] that under these hypotheses, $X$ has a subspace isomorphic to $l^{1}$. Their proof is related to a construction of $\mathrm{A}$. Nissenzweig [41]. (Of course Theorem 3 shows that the answer is affirmative if $X$ is isomorphic to $Y^{*}$ for some separable $Y$.) Suppose we just assume the weaker hypothesis

$X^{*}$ has a bounded sequence with no weak* convergent subsequence.

Then if $c_{0}$ is not isomorphic to a quotient space of $X$, the results of [32] and [51] again imply that the stronger hypotheses of the question hold, hence $l^{1}$ embeds in $X$. On the other hand, J. Hagler and E. Odell [24] have constructed a space $X$ containing no isomorph of $l^{1}$ yet satisfying (*). In earlier work, $\mathrm{R}$. Haydon [25] constructed a compact Hausdorff space $S$ so that $X=C(S)$ satisfies (*) yet contains no isomorph of $l^{\prime}(\Gamma)$ for any uncountable set $\Gamma$. If $X$ satisfies (*), then Johnson and Hagler also show in [23] that $X$ has a separable subspace with a nonseparable dual.

There seems to be no known criterion similar to the failure of 4 , for characterizing Banach spaces containing an isomorph of $l^{1}(\Gamma)$ for some uncountable $\Gamma$. Both Hagler [21] and Haydon [26] have constructed spaces $X$ containing no isomorph of $l^{1}(\Gamma)$ for any uncountable $\Gamma$, yet containing an uncountable set in which there is no weak-Cauchy sequence. Hagler generalized the implication $1 \Rightarrow 11$ in [20] by showing that for general $B, l^{1}$ 
embeds in $B$ provided $l^{1}(\Gamma)$ embeds in $B^{*}$ for some set $\Gamma$ with $\operatorname{dim} B<$ card $\Gamma$, where $\operatorname{dim} B$ is the smallest cardinality of a subset of $B$ with dense linear span. Haydon obtained a strong nonseparable analogue for the equivalence of 1 and 10 in [26]. He showed that if $\tau$ is a regular cardinal number so that $\mathfrak{N}^{\omega}<\tau$ whenever $\Re<\tau$, then $l^{1}(\tau)$ embeds in $B$ if and only if $L^{1}\left(\{0,1\}^{+}\right)$embeds in $B^{*}$. Haydon also obtains a kind of generalization of the criterion in 3 to nonseparable $B$ in [25]. Let $K$ denote the unit ball of $B^{*}$ in its weak*-topology. 3 implies that every element of $B^{* *}$ is Baire-1 on $K$ and hence Borel measurable on $K$, assuming $B$ is separable. For general $B$, a function $\phi$ on $K$ is said to be universally measurable provided it is measurable with respect to the completion of any regular Borel probability measure on $K$. Haydon's criterion then asserts that $l^{1}$ does not embed in $B$ if and only if every element of $B^{* *}$ is universally measurable on $K$.

2. Sequences of functions with no point-wise convergent subsequence. Theorem 1 of $\$ 1$ follows immediately from the following result:

THEOREM 2.1. Let $S$ be a set and $\left(f_{j}\right)$ a uniformly bounded sequence of scalar valued functions on $S$ with no point-wise convergent subsequence. Then $\left(f_{j}\right)$ has a subsequence $\left(f_{j}^{\prime}\right)$ so that there is a $\delta>0$ with $\sup _{s \in S}\left|\sum_{j=1}^{n} c_{j} f_{j}^{\prime}(s)\right|>$ $\delta \sum_{j=1}^{n}\left|c_{j}\right|$ for every $n$ and scalars $c_{1}, \ldots, c_{n}$.

To deduce Theorem 1, let $B$ be a Banach space and let $\left(b_{j}\right)$ be a bounded sequence in $B$ with no weak-Cauchy subsequence. Let $S$ be the unit ball of $B^{*}$ and define $f_{j}(s)=s\left(b_{j}\right)$ for all $j$ and $s \in S$. Then $\left(f_{j}\right)$ is uniformly bounded with no point-wise convergent subsequence; choosing $\left(f_{j}^{\prime}\right)$ and $\delta$ as in 2.1 and letting $\left(b_{j}^{\prime}\right)$ be the corresponding sequence in $B$, we have that

$$
\left\|\sum_{j=1}^{n} c_{j} b_{j}^{\prime}\right\| \geqslant \sup _{s \in S}\left|\sum_{j=1}^{n} c_{j} f_{j}^{\prime}(s)\right| \geqslant \delta \sum_{j=1}^{n}\left|c_{j}\right|
$$

for every $n$ and scalars $c_{1}, \ldots, c_{n}$, thus proving Theorem 1 .

We will discuss 2.1 for the case of real scalars. See [11] for the complex scalars case.

Theorem 2.1 frees us from thinking about general Banach spaces; we just consider sequences of functions defined on some set. We can try to make the functions as simple as possible, to see what is happening. If the functions are 1-valued, i.e. constants, then nothing is happening; a bounded sequence of constants has a convergent subsequence. However suppose the functions $f_{1}, f_{2}, \ldots$ are two-valued, say $\{1,-1\}$-valued. Then a great deal is happening and the fundamental idea of the proof of Theorem 2.1 is revealed.

Let $A_{n}=\left\{x \in S: f_{n}(x)=1\right\}$ and $B_{n}=\left\{x \in S: f_{n}(x)=-1\right\}$. It is easily seen that $\left(f_{n}\right)$ converges point-wise if and only if $\left(A_{n}\right)$ converges, where $\left(A_{n}\right)$ is said to converge provided $\left(\chi_{A_{A}}\right)$ converges pointwise $\left(\chi_{A}\right.$ denotes the characteristic function of the set $A$ ). On the other hand, suppose the $A_{n}$ 's are Boolean independent, that is, for any disjoint finite subsets $F$ and $G$ of indices, $\cap_{n \in F} A_{n} \cap \cap_{n \in G} B_{n} \neq \phi$. (We make the convention that if $F$ is empty, $\cap_{n \in F} A_{n}=S$.) Then it is easily seen that the $f_{n}$ 's are isometrically equivalent to the usual $l^{1}$-basis in the supremum norm. Indeed, given real scalars $c_{1}, \ldots, c_{n}$, let $F=\left\{i: c_{i}>0\right\}$ and $G=\left\{i: c_{i} \leqslant 0\right\}$. Choose $t \in$ 


$$
\begin{aligned}
& \cap_{n \in G} A_{n} \cap \cap_{n \in G} B_{n} \text {. Then } \\
& \qquad \begin{aligned}
\sum_{i=1}^{n} c_{i} f_{i}(t) & =\sum_{i \in F} c_{i} f_{i}(t)+\sum_{i \in G} c_{i} f_{i}(t) \\
& =\sum_{i \in F} c_{i}+\sum_{i \in G}-c_{i}=\sum_{i=1}^{n}\left|c_{i}\right| .
\end{aligned}
\end{aligned}
$$

Hence $\sup _{t \in S}\left|\sum_{i=1}^{n} c_{i} f_{i}(t)\right|=\sum_{i=1}^{n}\left|c_{i}\right|$. The 2-valued case for Theorem 2.1 thus follows from the following combinatorial result in set theory, discovered by the author in [51]: THEOREM. Let $\left(A_{n}\right)$ be a sequence of sets with no convergent subsequence. Then $\left(A_{n}\right)$ has a Boolean independent subsequence.

We note in passing that the so-called Rademacher sequence $f_{n}(x)=$ sign $\sin 2^{n} \pi x$ is perhaps the generic example of a sequence equivalent in the supremum norm to the usual $l^{1}$-basis. In this case the Boolean independence of the corresponding $A_{n}$ 's is a consequence of their statistical independence.

We require a slight generalization of the above Theorem, in order to derive our combinatorial proof of Theorem 2.1. It is also convenient to introduce the following terminology: By a sequence we shall mean a set of objects indexed by some infinite subset $M$ of the positive integers $N$. We shall understand by "a subset of $M$ " an infinite subset of $M$, unless the contrary is explicitly stated. Given $L$ and $M$ subsets of $N$, say that $L$ is almost contained in $M$ (notation: $L \subset_{a} M$ ) if $L \cap \sim M$ is a finite set, (" $\sim M$ " denotes the complement of the set $M$ ). Given a sequence $\left(f_{n}\right)_{n \in M}$ and $L$ and $Q$ subsets of $M$ with $L \subset_{a} Q$, we call $\left(f_{n}\right)_{n \in L}$ a subsequence of $\left(f_{n}\right)_{n \in Q}$. We now need to generalize the notions of convergence and independence of sequences of sets to sequences of pairs of sets.

DEFINITION. Let $\left(A_{n}, B_{n}\right)_{n \in M}$ be a sequence of pairs of subsets of the set $S$ with $A_{n} \cap B_{n}=\varnothing$ for all $n$ and let $X$ be a subset of $S$. We say that $\left(A_{n}, B_{n}\right)_{n \in M}$ converges if every point $s \in S$ belongs to at most finitely many $A_{n}$ 's or finitely many $B_{n}$ 's. We say that $\left(A_{n}, B_{n}\right)_{n \in M}$ converges on $X$ if $\left(A_{n} \cap X, B_{n} \cap X\right)_{n \in M}$ converges. We say that $\left(A_{n}, B_{n}\right)_{n \in M}$ is independent if for every pair of disjoint finite subsets $F$ and $G$ of $M$,

$$
\bigcap_{n \in F} A_{n} \cap \bigcap_{n \in G} B_{n} \neq \varnothing \text {. }
$$

Note that in the special case $B_{n}=S \sim A_{n}$ for all $n,\left(A_{n}, B_{n}\right)$ converges on $X$ if and only if $\chi_{A_{n}}$ converges point-wise on $X$. The generalization of the above Theorem now goes as follows:

THEOREM 2.2. Let $\left(A_{n}, B_{n}\right)_{n \in N}$ be a sequence of pairs of subsets of $S$ witn $A_{n} \cap B_{n}=\varnothing$ for all $n$, and suppose $\left(A_{n}, B_{n}\right)_{n \in N}$ has no convergent subsequence. Then $\left(A_{n}, B_{n}\right)_{n \in N}$ has an independent subsequence.

J. Farahat [17] has derived this result from a combinatorial result of $F$. Galvin and K. Prikry [19] concerning Ramsey subsets of $N$ (based on earlier work of C. St. J. A. Nash-Williams [40]; see also [14]). We prefer, however, to go over the original ideas of the proof given in [51]. Before doing so, we shall deduce 2.1 from 2.2 .

Proposition 2.3. Let $\left(f_{n}\right)$ be a sequence of real-valued functions on $S$ and $r$, 
$\delta$ real numbers with $\delta>0$ so that $\left(A_{n}, B_{n}\right)_{n \in N}$ is independent, where

$$
A_{n}=\left\{s \in S: f_{n}(s)>r+\delta\right\}
$$

and

$$
B_{n}=\left\{s \in S: f_{n}(s)<r\right\} \text { for all } n .
$$

Then for any $n$ and scalars $c_{1}, \ldots, c_{n}$,

$$
\sup _{s \in S}\left|\sum_{j=1}^{n} c_{j} f_{j}(s)\right| \geqslant \frac{\delta}{2} \sum_{j=1}^{n}\left|c_{j}\right| .
$$

(Consequently if $\left(f_{j}\right)$ is uniformly bounded it is equivalent in the supremum norm to the usual $l^{1}$-basis.)

Proof. We adopt the convention that the sum of an empty family of scalars is 0 ; i.e. $\Sigma_{j \in \phi} d_{j}=0$. Fix $n$, let $c_{1}, \ldots, c_{n}$ be real scalars, and let $\mathscr{P}=\left\{i: c_{i}>0\right\}$ and $\mathscr{\Re}=\left\{i: c_{i} \leqslant 0\right\}$. By the independence of $\left(A_{j}, B_{j}\right)_{j \in N}$ we may choose $x \in \cap_{j \in \mathcal{P}} A_{j} \cap \cap_{j \in \Re} B_{j}$ and $y \in \bigcap_{j \in \Re} A_{j} \cap \bigcap_{j \in \mathcal{P}} B_{j}$. We then have

$$
\begin{aligned}
\sum_{j \in \mathscr{T}} c_{j} f_{j}(x) & \geqslant \sum_{j \in \mathscr{P}}\left|c_{j}\right|(r+\delta) \\
\sum_{j \in \mathscr{\Re}} c_{j} f_{j}(x) & =\sum_{j \in \mathscr{\Re}}-c_{j}\left(-f_{j}(x)\right) \geqslant \sum_{j \in \mathscr{\Re}}\left|c_{j}\right|(-r) \\
-\sum_{j \in \mathscr{P}} c_{j} f_{j}(y) & =\sum_{j \in \mathscr{P}} c_{j}\left(-f_{j}(y)\right) \geqslant \sum_{j \in \mathscr{P}}\left|c_{j}\right|(-r) \\
-\sum_{j \in \Re} c_{j} f_{j}(y) & \geqslant \sum_{j \in \Re}\left|c_{j}\right|(r+\delta) .
\end{aligned}
$$

Adding the inequalities (1)-(4), we obtain

$$
\sum_{j=1}^{n} c_{j} f_{j}(x)-\sum_{j=1}^{n} c_{j} f_{j}(y) \geqslant\left(\sum_{j=1}^{n}\left|c_{j}\right|\right) \delta .
$$

Proposition 2.3 follows from (5) and the observation that

$$
\begin{aligned}
\sup _{s \in S}\left|\sum_{j=1}^{n} c_{j} f_{j}(s)\right| & \geqslant \max \left\{\left|\sum_{j=1}^{n} c_{j} f_{j}(x)\right|,\left|\sum_{j=1}^{n} c_{j} f_{j}(y)\right|\right\} \\
& \geqslant \frac{1}{2}\left(\sum_{j=1}^{n} c_{j} f_{j}(x)-\sum_{j=1}^{n} c_{j} f_{j}(y)\right) .
\end{aligned}
$$

We require one more result to obtain Theorem 2.1 from Theorem 2.2.

LEMMA 2.4. Let $\left(f_{j}\right)_{j \in N}$ be a point-wise bounded sequence of real-valued functions defined on $S$ and having no point-wise convergent subsequence. Then there exist an $N^{\prime} \subset N$, and real numbers $r$ and $\delta$ with $\delta>0$ so that for all $M \subset N^{\prime}$, there is an $s \in S$ with

$$
\begin{gathered}
f_{m}(s)>r+\delta \text { for infinitely many } m \in M \text { and } \\
f_{m}(s)<r \text { for infinitely many } m \in M .
\end{gathered}
$$


To deduce Theorem 2.1, let $\left(f_{j}\right)_{j \in N}$ be as in its statement. Choose $N^{\prime} \subset N$ and $r$ and $\delta$ with $\delta>0$ satisfying (6) of Lemma 2.4 for all $M \subset N^{\prime}$. It follows that if $A_{n}=\left\{s \in S: f_{n}(s)>r+\delta\right\}$ and $B_{n}=\left\{s \in S: f_{n}(s)<r\right\}$ for all $n \in N^{\prime}$, then $\left(A_{n}, B_{n}\right)_{n \in N^{\prime}}$ has no convergent subsequence. By 2.2 there exists an $N^{\prime \prime} \subset N^{\prime}$ with $\left(A_{n}, B_{n}\right)_{n \in N^{\prime \prime}}$ independent. Hence by Proposition 2.3, $\left(f_{n}\right)_{n \in N^{\prime \prime}}$ satisfies the conclusion of Theorem 2.1.

PROOF OF LEMMA 2.4. For $M \subset N$ and $s \in S$ we set $\lim _{i n f} f_{m \in M} f_{m}(s)=$ $\lim \inf _{j \rightarrow \infty} f_{m_{j}}(s)$ and $\lim \sup _{m \in M} f_{m}(s)=\lim \sup _{j \rightarrow \infty} f_{m_{i}}(s)$ where $\left\{m_{1}, m_{2}, \ldots\right\}$ is a strictly increasing enumeration of $M$. Let $\left(r_{1}, \delta_{1}\right)$, $\left(r_{2}, \delta_{2}\right), \ldots$ be an enumeration of all pairs of rational numbers $(r, \delta)$ with $\delta>0$. Say that $N^{\prime} \subset N, r, \delta$ work if they satisfy the conclusion of the Lemma. Suppose nothing works. Since $N, r_{1}$, and $\delta_{1}$ don't work, we may choose $M_{1} \subset N$ so that (6) fails for all $s \in S$ and " $M "=M_{1}, " r "=r_{1}$ and " $\delta$ " = $\delta_{1}$. Having chosen $M_{n} \subset N$, since $M_{n}, r_{n+1}$ and $\delta_{n+1}$ don't work, we may choose $M_{n+1} \subset M_{n}$ so that (6) fails for all $s \in S$ and " $M "=M_{n+1}$, " $r "=r_{n+1}$ and " $\delta "=\delta_{n+1}$. Now by the Cantor diagonalization procedure, we may select an $M \subset N$ with $M$ almost contained in $M_{n}$ for all $n$. Then $\left(f_{j}\right)_{j \in M}$ converges point-wise. Indeed, if not, by point-wise boundedness of $\left(f_{j}\right)$ we may choose an $s \in S$ and rational numbers $r, \delta$ with $\delta>0$ so that

$$
\liminf _{m \in M} f_{m}(s)<r<r+\delta<\limsup _{m \in M} f_{m}(s) .
$$

Now choose $n$ so that $(r, \delta)=\left(r_{n}, \delta_{n}\right)$. Since $M \subset_{a} M_{n}$ we have that

$$
\liminf _{m \in M_{n}} f_{m}(s)<r_{n}<r_{n}+\delta_{n}<\limsup _{m \in M_{n}} f_{m}(s) \text {. }
$$

But by construction of the $M_{j}$ 's, $\lim \sup _{m \in M_{n}} f_{m}(s) \leqslant r_{n}+\delta_{n}$ or $\lim \inf _{m \in M_{n}} f_{m}(s) \geqslant r_{n}$, contradicting (8). Hence $\left(f_{j}\right)_{j \in N}$ has a point-wise convergent subsequence, contradicting our initial assumptions and completing the proof of the lemma.

We now pass to the proof of Theorem 2.2. For the remainder of this discussion, $\left(A_{n}, B_{n}\right)_{n \in N}$ shall denote a sequence of pairs of subsets of $S$ with $A_{n} \cap B_{n}=\varnothing$ for all $n$. It is easy to verify the following permanence properties of convergence of such sequences: if $L \subset M \subset N, X \subset S$, and $\left(A_{j}, B_{j}\right)_{j \in N_{1}}$ converges on $A_{n_{1}}$; otherwise set $\varepsilon_{n_{1}}=-1$. Suppose $k>1$ and converges on $X$, it converges on $Y$. If $X_{1}, X_{2}, \ldots$ are subsets of $S$ and $X=\cup_{i=1}^{\infty} X_{i},\left(A_{n}, B_{n}\right)_{n \in M}$ converges on $X$ if it converges on $X_{i}$ for all $i$. Finally, $\left(A_{n}, B_{n}\right)_{n \in N}$ converges on the empty set. We introduce the following notation: $+A_{n}=A_{n}$ and $-A_{n}=B_{n}$ for all $n$. For $j \in N$ and $M \subset N$, we say that $j$ and $M$ work provided $\left(A_{n}, B_{n}\right)_{n \in M}$ has no subsequence convergent on either $A_{j}$ or $B_{j}$. For the first step of the proof, we shall exhibit a kind of constructive procedure which shows that if nothing works, $\left(A_{n}, B_{n}\right)_{n \in N}$ has a convergent subsequence.

Let $n_{1} \in N$ be arbitrary. Suppose $n_{1}$ and $N$ don't work. Then we may choose $N_{1} \subset N$ so that $\left(A_{j}, B_{j}\right)_{j \in N_{1}}$ converges on $A_{n_{1}}$ or $B_{n_{1}}$. Set $\varepsilon_{n_{1}}=+1$ if $\left(A_{j}, B_{j}\right)_{j \in N_{1}}$ converges on $A_{n_{1}}$; otherwise set $\varepsilon_{n_{1}}=-1$. Suppose $k>1$ and $n_{k-1}$ and $N_{k-1} \subset N$ have been chosen. Let $n_{k} \in N_{k-1}$ with $n_{k}>n_{k-1}$. Suppose $n_{k}$ and $N_{k-1}$ don't work. Then choose $N_{k} \subset N$ so that $\left(A_{j}, B_{j}\right)_{j \in N_{k}}$ converges on $A_{n_{k}}$ or $B_{n_{k}}$; set $\varepsilon_{n_{k}}=+1$ if the former happens, otherwise set 
$\varepsilon_{n_{k}}=-1$. Suppose we can continue this procedure indefinitely. Then setting $M=\left\{n_{1}, n_{2}, \ldots\right\}$, we have that for each $k,\left(A_{j}, B_{j}\right)_{j \in M}$ is a subsequence of $\left(A_{j}, B_{j}\right)_{j \in N_{k}}$ which converges on $\left\{\varepsilon_{n_{k}} A_{n_{k}}\right\}$. Hence $\left(A_{j}, B_{j}\right)_{j \in M}$ converges on $\cup_{k=1}^{\infty} \varepsilon_{n_{k}} A_{n_{k}}$. Now choose $M^{\prime} \subset M$ infinite so that either

(i) $\varepsilon_{m}=+1$ for all $m \in M^{\prime}$ or

(ii) $\varepsilon_{m}=-1$ for all $m \in M^{\prime}$.

We claim that $\left(A_{j}, B_{j}\right)_{j \in M^{\prime}}$ converges. Indeed, supposing (ii) holds, we have that $\left(A_{j}, B_{j}\right)_{j \in M^{\prime}}$ converges on $\bigcup_{m \in M^{\prime}} \varepsilon_{m} A_{m}=\cup_{m \in M^{\prime}} B_{m}$, since $\bigcup_{m \in M^{\prime}} \varepsilon_{m} A_{m} \subset \cup_{k=1}^{\infty} \varepsilon_{n_{k}} A_{n_{k}}$. Hence if $s \in S$ belongs to infinitely many $B_{j}$ 's for $j \in M^{\prime}, s \in \cup_{m \in M^{\prime}} B_{m}$ so $s$ belongs to only finitely many $A_{j}$ 's for $j \in M^{\prime}$. Thus every $s$ belongs to at most finitely many $A_{j}$ 's or at most finitely many $B_{j}$ 's for $j \in M^{\prime}$. The proof supposing (i) is the same. We may summarize what we have proved as follows:

LEMmA 2.5. Suppose there exist $N=N_{0} \supset N_{1} \supset N_{2} \supset \ldots$ and $n_{1}<n_{2}$ $<\ldots$ so that for all $k \geqslant 1, n_{k} \in N_{k-1}$ and $\left(A_{j}, B_{j}\right)_{j \in N_{k}}$ converges on either $A_{n_{k}}$ or $B_{n_{k}}$. Then $\left(A_{j}, B_{j}\right)_{j \in N}$ has a convergent subsequence.

We may now easily deduce the following result by induction:

LemMA 2.6. Let $l \geqslant 1, X_{1}, \ldots, X_{l}$ disjoint subsets of $S, N^{\prime} \subset N$, and suppose that for each $i, 1 \leqslant i \leqslant l,\left(A_{n}, B_{n}\right)_{n \in N^{\prime}}$ has no subsequence convergent on $X_{i}$. Then there exist a $j \in N^{\prime}$ and an $M \subset N^{\prime}$ so that for each $i, 1 \leqslant i \leqslant l$, $\left(A_{n}, B_{n}\right)_{n \in M}$ has no subsequence convergent on $A_{j} \cap X_{i}$ or $B_{j} \cap X_{i}$.

PROOF. Suppose this has been proved for $l-1$ (in case $l>1$ ). Say that for $r \geqslant 0, j$ and $M \subset N r$-work provided $r=0$ or $r \geqslant 1$ and $\left(A_{n}, B_{n}\right)_{n \in M}$ has no subsequence convergent on $A_{j} \cap X_{i}$ or $B_{j} \cap X_{i}$ for all $1 \leqslant i \leqslant r$. Note that if $j$ and $M l-1$-work, then $j$ and $M$ don't $l$-work if and only if $\left(A_{n} \cap X_{l}\right.$, $\left.B_{n} \cap X_{l}\right)_{n \in M}$ has a subsequence converging on either $A_{j} \cap X_{l}$ or $B_{j} \cap X_{l}$. Choose $n_{1}$ and $N_{1}^{\prime} \subset N^{\prime}$ so that $n_{1}$ and $N_{1}^{\prime} l-1$-work. By discarding a finite subset of $N_{1}^{\prime}$ if necessary, we may assume $n_{1}<n$ all $n \in N_{1}^{\prime}$. If $n_{1}$ and $N_{1}^{\prime}$ don't l-work, choose $N_{1} \subset N_{1}^{\prime}$ so that $\left(A_{n}, B_{n}\right)_{n \in N_{1}}$ converges on either $A_{n_{1}} \cap X_{l}$ or $B_{n_{1}} \cap X_{l}$. Suppose $k>1$ and $n_{k-1}$ and $N_{k-1} \subset N$ have been chosen with $n_{k-1}<n$ for all $n \in N_{k-1}$. Since $\left(A_{j}, B_{j}\right)_{j \in N_{k-1}}$ has no convergent subsequence, by the induction hypothesis there is an $N_{k}^{\prime} \subset N_{k-1}$ and an $n_{k} \in N_{k-1}$ so that $n_{k}$ and $N_{k}^{\prime} l-1$-work. Again we may assume that $n_{k}<n$ for all $n \in N_{k}^{\prime}$. So if $n_{k}$ and $N_{k}^{\prime}$ don't $l$-work, choose $N_{k} \subset N_{k}^{\prime}$ so that $\left(A_{n}, B_{n}\right)_{n \in N_{k}}$ converges on either $A_{n_{k}} \cap X_{l}$ or $B_{n_{k}} \cap X_{l}$. We cannot continue this process indefinitely, for otherwise $\left(A_{j} \cap X_{l}, B_{j} \cap X_{l}\right)_{j \in N^{\prime}}$ would have a convergent subsequence by the preceding Lemma. Hence for some $k, n_{k}$ and $N_{k}^{\prime} l$-work, completing the proof.

Proof of Theorem 2.2. Assume $\left(A_{j}, B_{j}\right)_{j \in N}$ has no convergent subsequence. We may then choose $n_{1}$ and $N_{1} \subset N$ so that $n>n_{1}$ for all $n \in N_{1}$ and $\left(A_{j}, B_{j}\right)_{j \in N_{1}}$ has no subsequence convergent on $A_{n_{1}}$ or $B_{n_{j}}$. Suppose $k>1$ and $n_{1}<n_{2}<\cdots<n_{k}$ and $N_{k} \subset N$ have been constructed so that $n>n_{k}$ for all $n \in N_{k}$ and for each $\left(\varepsilon_{1}, \ldots, \varepsilon_{k}\right)$ with $\varepsilon_{i}= \pm 1$ for all $i$, $\left(A_{j}, B_{j}\right)_{j \in N_{k}}$ has no subsequence convergent on $\cap_{i=1}^{k} \varepsilon_{i} A_{n_{i}}$. By Lemma 2.6 there exist $N_{k+1} \subset N_{k}$ and $n_{k+1} \in N_{k}$ so that $\left(A_{j}, B_{j}\right)_{j \in N_{k+1}}$ has no subsequence convergent on $\left(\cap_{i=1}^{k} \varepsilon_{i} A_{n_{i}}\right) \cap A_{n_{k+1}}$ or $\left(\bigcap_{i=1}^{k} \varepsilon_{i} A_{n_{i}}\right) \cap B_{n_{k+1}}$. Of 
course, we may assume $n>n_{k+1}$ for all $n \in N_{k+1}$. It now follows that $\left(A_{n_{i}}, B_{n_{i}}\right)_{i=1}^{\infty}$ is independent, since $\bigcap_{i=1}^{k} \varepsilon_{i} A_{n_{i}} \neq \varnothing$ for all $k$ and $\left(\varepsilon_{1}, \ldots, \varepsilon_{k}\right)$ with $\varepsilon_{i}= \pm 1$ for all $i$.

The complex-scalars case of Theorem 2.1 is deduced from Theorem 2.3 in [11] by suitable complex analogues of Proposition 2.3 and Lemma 2.4 above. The analogue of Proposition 2.3 asserts that a uniformly bounded sequence $\left(f_{n}\right)_{n \in N}$ is equivalent in the supremum norm to the usual $l^{1}$-basis over the complex scalars provided there exists a pair of open discs $\left(D_{1}, D_{2}\right)$ in the complex plane with equal diameters smaller than one half the distance between them so that $\left(A_{n}, B_{n}\right)_{n \in N}$ is independent, where $A_{n}=\left\{s: f_{n}(s) \in\right.$ $\left.D_{1}\right\}$ and $B_{n}=\left\{s: f_{n}(s) \in D_{2}\right\}$ for all $n$. The analogue of 2.4 asserts that if $\left(f_{n}\right)$ has no point-wise convergent sequence and is uniformly bounded, then it has a subsequence $\left(f_{n}\right)_{n \in M}$ for which there exist such discs $D_{1}$ and $D_{2}$ so that $\left(A_{n}, B_{n}\right)_{n \in M}$ has no convergent subsequence. For details, see [11] and also pp. 99-101 of [38].

3. Point-wise compact subsets of the first Baire class. This section is devoted to a discussion of Theorem 2 of $\S 1$. We first require some definitions. Let $X$ be a topological space. A real-valued function $f$ on $X$ is said to belong to the first Baire class on $X$ provided there exists a sequence of real-valued continuous functions on $X$ which converges to $f$ point-wise. We let $B_{1}(X)$ denote the family of all Baire-1 functions on $X$ endowed with the topology of point-wise convergence; that is, a net $\left(f_{\alpha}\right)_{\alpha \in D}$ of elements of $B_{1}(X)$ converges to an $f \in B_{1}(X)$ if and only if $\lim _{\alpha \in D} f_{\alpha}(x)=f(x)$ for all $x \in X$. We also note the following form of the definition of the topology of point-wise convergence: Given $G$ a family of real-valued functions on $X$, then a function $f$ on $X$ is in the point-wise closure of $G$ if and only if for every $\varepsilon>0$ and finite number of points $x_{1}, \ldots, x_{n}$ in $X$, there is a $g \in G$ with $\left|g\left(x_{i}\right)-f\left(x_{i}\right)\right|$ $<\varepsilon$ for all $1 \leqslant i \leqslant n$. We recall finally that $X$ is said to be a Polish space if $X$ is homeomorphic to a complete separable metric space.

The result we wish to discuss is due to J. Bourgain, D. H. Fremlin and M. Talagrand [7], based on earlier work of the author's [54]. It goes as follows:

Theorem 3.1. Let $X$ be a Polish space and $F$ be a subset of $B_{1}(X)$ so that every countable subset of $F$ has a cluster point in $B_{1}(X)$. Then $F$ is relatively compact and sequentially dense in its closure. That is, the set of all limits of point-wise convergent sequences of elements of $F$ forms a point-wise compact subset of the first Baire class.

Certainly the depth of this result is contained in the case where $X=[0,1]$; indeed, most of the applications to Banach space theory can be deduced from this case. The result is not stated in the context of arbitrary Polish spaces just for the sake of generality, however. One of the steps in the proof involves the introduction of a finer Polish topology on $X$; so if $X=[0,1]$, it still seems necessary to introduce a noncompact Polish topology on $[0,1]$ in order to complete the proof.

We shall first discuss some immediate consequences of the Theorem.

Corollary 3.2. When $X$ is a Polish space, a relatively compact subset of $B_{1}(X)$ is sequentially compact. 
Indeed, let $F$ be relatively compact and $\left(f_{n}\right)$ a sequence of distinct elements of $F$. Since $F$ is relatively compact, there exists an $f \in B_{1}(X)$ which is a cluster point of $\left\{f_{n}: n=1,2, \ldots\right\}$. But then some subsequence of $\left(f_{n}\right)$ converges to $f$. Corollary 3.2 is due to the author, and is in fact required as one of the steps of the proof of Theorem 3.1 (see Lemma 3.8 below).

This corollary shows that Theorem 3.1 may be viewed as a generalization of the Helly selection theorem. Indeed, let $H$ denote the family of nondecreasing functions mapping the unit interval $[0,1]$ into itself. It is easily seen that $H$ is a point-wise compact subset of $B_{1}([0,1])$. Thus the corollary implies that any sequence $\left(f_{n}\right)$ in $H$ has a point-wise convergent subsequence; this is essentially the Helly selection theorem.

Next, let us deduce Theorem 2 of $\S 1$. Suppose $B$ is a separable Banach space containing no isomorph of $l^{1}$ and let $X$ denote the unit ball of $B^{*}$ endowed with the weak ${ }^{*}$-topology. Then $X$ is a compact metrizeable space, hence is Polish. Every element of $B$, regarded as a subset of $B^{* *}$, is a continuous function on $X$. Now let $F$ denote the unit ball of $B$ and $G$ the unit ball of $B^{* *}$ endowed with the weak*-topology. Theorem 1 implies that every $\left(b_{n}\right)$ in $F$ has a weak-Cauchy subsequence $\left(b_{n}^{\prime}\right)$. Defining $f(x)=\lim _{n \rightarrow \infty} x\left(b_{n}^{\prime}\right)$ for all $x \in X, f$ is Baire- 1 on $X$, hence $F$ is a relatively compact subset of $B_{1}(X)$ by Theorem 3.1. Goldstine's theorem asserts that $G$ equals the pointwise closure of $F$, hence $G$ is a compact subset of $B_{1}(X)$. Thus by 3.1, every subset of $G$ is sequentially dense in its point-wise closure, which implies Theorem 2 of $\$ 1$.

The above argument yields immediately a stronger version of Theorem 2 (in view of Theorem 1). Say that a subset of a Banach space is weakly pre-compact if every sequence in the subset has a weak-Cauchy subsequence. The uniform boundedness principle implies that a weakly pre-compact set is bounded in the norm topology. We then have:

COROLlaRy 3.3. Let $F$ be a weakly pre-compact subset of a separable Banach space $B$. Then every subset of the weak ${ }^{*}$-closure of $F$ in $B^{* *}$ is sequentially dense in its weak*-closure. In particular, $F$ is weakly sequentially dense in its weak closure.

We note in passing that if $B$ is separable and $X$ is as in the proof of Theorem 2 above, then a result of E. Odell and the author [42] asserts that if $h \in B^{* *}$ is Baire-1 on $X$, there exists a sequence $\left(b_{n}\right)$ in $B$ with $b_{n} \rightarrow h$ weak $^{*}$ as $n \rightarrow \infty$. It follows that the subsets of $B^{* *}$ which are point-wise compact subsets of $B_{1}(X)$ coincide with the weak ${ }^{*}$-compact subsets of $B^{* *}$ which are contained in the sequential closure of $B$ in $B^{* *}$. (By the sequential closure of $B$ we mean the set of all elements of $B^{* *}$ which are the weak*-limit of some weak-Cauchy sequence in $B$.) This suggests the following question: Let $B$ be $a$ separable Banach space and $G$ be a weak ${ }^{*}$-compact subset of $B^{* *}$ contained in the sequential closure of $B$. Does there exist a weakly pre-compact set $F$ in $B$ so that $G$ is contained in the weak ${ }^{*}$-closure of $F$ ?**

We give one last immediate corollary, proved by the author in [54] using cumbersome techniques.

\footnotetext{
${ }^{* *}$ ADDED IN PROOF. This question has been answered in the negative by J. Bourgain in a paper entitled Some remarks on compact sets of the first Baire class (to appear).
} 
Corollary 3.4. Let $X$ be a Polish space and $F$ a uniformly bounded relatively compact subset of $B_{1}(X)$. Let $\left(f_{\alpha}\right)_{\alpha \in D}$ be a point-wise convergent net of elements of $F$ with limit $f$. Then $f$ is Borel measurable and $\int f_{\alpha} d \mu \rightarrow \int f d \mu$ for all signed Borel measures $\mu$ on $X$.

The proof (as given in [7]) goes as follows: Let $G$ denote the closure of $F$ in $B_{1}(X)$. Then $f \in G$ and every element of $G$ is Borel-measurable, being Baire-1 on $X$. Define a function $\phi: G \rightarrow \mathbf{R}$ by $\phi(g)=\int g d \mu$ for all $g \in G$. It suffices to show that $\phi$ is continuous. Now $\phi$ is sequentially continuous by the bounded convergence theorem. Theorem 3.1 then implies that $\phi$ is continuous. Indeed, let $\boldsymbol{A}$ be a closed subset of $\mathbf{R}$, the real numbers. If $g$ belongs to the closure of $\phi^{-1}(A)$, then Theorem 3.2 shows that there exists a sequence $\left(g_{n}\right)$ in $\phi^{-1}(A)$ with $g_{n} \rightarrow g$. But then $\phi\left(g_{n}\right) \rightarrow \phi(g)$, so $\phi(g) \in A$ and thus $g \in \phi^{-1}(A)$, i.e. $\phi^{-1}(A)$ is closed.

Before passing to a detailed discussion of the proof of Theorem 3.1, we would just like to mention the following deep stability property of point-wise compact subsets of the first Baire class, due to Bourgain, Fremlin and Talagrand [7]:

Theorem. Let $X$ be a Polish space and $F$ a uniformly bounded relatively compact subset of $B_{1}(X)$. Then the convex hull of $F$ is also relatively compact in $B_{1}(X)$.

This result immediately gives the following corollary, due to C. Stegall: Let $F$ be a weakly pre-compact subset of some Banach space. Then the convex hull of $F$ is also weakly precompact. Stegall's proof used Theorem 1 of $\$ 1$. For a discussion of a related argument, see the addendum of [54].

We pass now to the proof of Theorem 3.4. The demonstration lies far below the surface, and makes use of topological reasoning and transfinite induction. We break the proof into ten steps, giving the key ideas in the demonstration of each step. Those steps requiring several others or fairly involved arguments are called "Lemmas", while those with shorter proofs are termed "Scholiums". For detailed proofs of the various steps, see [7] and [54]. Throughout, we let $X$ denote a fixed Polish space, $N$ the natural numbers and $\omega_{1}$ the first uncountable ordinal. We follow the notations and conventions established in \$2. We shall make use of the following simple consequence of the Cantor diagonalization method:

if $M_{1}, M_{2}, \ldots$ are subsets of $N$ with $M_{n} \supset_{a} M_{n+1}$ for all $n$, then there exists a subset $M$ of $N$ with $M_{n} \supset_{a} M$ for all $n$.

We shall also make use of the following standard result: Given $\left\{K_{\alpha}\right.$ : $\left.\alpha<\omega_{1}\right\}$ with $K_{\alpha} \supset K_{\beta}$ and $K_{\alpha}$ a closed subset of $X$ for all $\alpha<\beta<\omega_{1}$, there exists an $\alpha_{0}<\omega_{1}$ with $K_{\alpha}=K_{\alpha_{0}}$ for all $\alpha>\alpha_{0}$. Phrased another way, there exists no strictly descending, uncountable transfinite sequence of closed subsets of a Polish space.

In a certain sense, the heart of the entire argument is contained in the "independently stated" Lemma 3.11, proved in [7], and occurring as a sort of "mid-point" of our discussion. The results preceding this lemma were proved in [54] and seem necessary to its proof. The reader already familiar with the 
earlier results of [54] may wish to pass directly to Lemma 3.11 and the discussion following it.

The overall proof of Theorem 3.1 makes crucial use of the following beautiful result published by R. Baire in 1899 [3] (see also [36] and pp. 288-289 of [27]):

LEMma 3.5 (THE BAIRe CHARACTERIZATION THEOREM). A real valued function $f$ defined on $X$ belongs to the first Baire class on $X$ if and only if for every nonempty closed subset $K$ of $X, f \mid K$ has a point of continuity relative to the topological space $K$.

Perhaps the simplest example of a non-Baire-1 function on the line is $f=\chi_{Q}$, where $Q$ denotes the set of rational numbers. The Baire characterization theorem allows us to say that any non-Baire-1 function on $X$ looks like $\chi_{Q}$ relative to some subset of $X$.

DefinITION. Let $f$ be a real-valued function defined on a topological space $Y$ and $L$ a subset of $Y$. We say that $f$ satisfies the Discontinuity Criterion on $L$ provided $L$ is nonempty and there are real numbers $r$ and $\delta$ with $\delta>0$ so that for every nonempty relatively open subset $U$ of $L$, there are $y$ and $z$ in $U$ with $f(y)>r+\delta$ and $f(z)<r$.

The first step in the proof of 3.1 follows from the Characterization Theorem and arguments in [42].

Scholium 3.6. A real-valued function $f$ defined on $X$ fails to belong to $B_{1}(X)$ if and only if $f$ satisfies the Discontinuity Criterion on some countable subset of $X$.

Proof. If $f$ satisfies the Discontinuity Criterion on $L$ then $f \mid \bar{L}$ has no points of continuity relative to $\bar{L}$, hence $f \notin B_{1}(X)$ by the Characterization Theorem. On the other hand, suppose $f \notin B_{1}(X)$. By the Characterization Theorem, choose a closed nonempty $K \subset X$ so that $f \mid K$ has no points of continuity relative to $K$. It follows that for every $k \in K$ there exist rational numbers $r$ and $\delta$ with $\delta>0$ so that

for every relatively open subset $U$ of $K$ with $k \in U$, there exist $y$ and $z$ in $U$ with $f(y)>r+\delta$ and $f(z)<r$.

Let $\left(r_{1}, \delta_{1}\right),\left(r_{2}, \delta_{2}\right), \ldots$ be an enumeration of all pairs of rational numbers $(r, \delta)$ with $\delta>0$. For each $n$, let $A_{n}=\left\{k \in K: k\right.$ satisfies (10) for $r=r_{n}$ and $\left.\delta=\delta_{n}\right\}$. Then $A_{n}$ is closed for all $n$ and $K=\cup_{n=1}^{\infty} A_{n}$. By the Baire category theorem there exists an $n$ so that $A_{n}$ has nonempty interior $V$ relative to $K$. It follows that $f$ satisfies the Discontinuity Criterion on $V$. Let $V_{1}, V_{2}, \ldots$ be a countable base for the relatively open subsets of $V$. For each $j$, choose $y_{j}$ and $z_{j}$ in $V_{j}$ with $f\left(y_{j}\right)>r+\delta$ and $f\left(z_{j}\right)<r$. Then setting $L=\left\{y_{j}, z_{j}: j=\right.$ $1,2, \ldots\}, f$ satisfies the Discontinuity Criterion on $L$.

REMARK. Using the above result, it is easily seen that if $f$ is not Baire-1 on $X$, there exists a compact totally disconnected perfect subset $K$ of $X$ so that $f \mid K$ is not Baire-1. That is, $f$ is not of the first Baire class relative to a subset of $X$ homeomorphic to the Cantor discontinuum. (This remark won't be used in the sequel.) 
We are now prepared for the first assertion of 3.1.

LEMMA 3.7. Let $F$ be a subset of $B_{1}(X)$ so that every countable subset of $F$ has a cluster point in $B_{1}(X)$. Then $F$ is relatively compact.

Proof. The hypotheses imply that for each $x \in X, M_{x}=\sup _{f \in F}|f(x)|<$ $\infty$. Now letting $\mathbf{R}^{X}$ denote the space of all real-valued functions on $X$ endowed with the topology of point-wise convergence, it follows that the closure of $F$ in $\mathbf{R}^{X}$, denoted by $G$, is compact. Indeed, $F \subset \Pi_{x \in X}\left[-M_{x}, M_{x}\right]$ which is compact by the Tychonoff theorem. Since the topology on $B_{1}(X)$ is simply the relative topology of $\mathbf{R}^{X}$ on $B_{1}(X)$, we need only show $G \subset B_{1}(X)$. Suppose $f \in G$ yet $f \notin B_{1}(X)$. By Scholium 3.6, there exists a countable set $L \subset X$ so that $f$ satisfies the Discontinuity Criterion on $L$. Since $L$ is countable and $f$ is a point-wise cluster point of $F$, there exists a sequence $\left(f_{n}\right)$ in $F$ so that $f_{n} \rightarrow f$ point-wise on $L$. But then if $g$ is a cluster-point of $\left(f_{n}\right)$ in $\mathbf{R}^{X}, g|L=f| L$, so by Scholium 3.6, $g \notin B_{1}(X)$, a contradiction.

The next step lies rather deeper; it is one of the main results in [54].

LeMma 3.8. $A$ compact subset of $B_{1}(X)$ is sequentially compact.

Proof. Let $F$ be a compact subset of $B_{1}(X)$ and $\left(f_{n}\right)$ a sequence in $F$. Suppose that $\left(f_{n}\right)$ has no point-wise convergent subsequence. We shall show that there exists a countable subset $L$ of $X$ and a subsequence $\left(f_{n}^{\prime}\right)$ of $\left(f_{n}\right)$ converging to a function $f$ satisfying the Discontinuity Criterion on $L$. Scholium 3.6 then implies $\left(f_{n}^{\prime}\right)$ has no Baire-1 cluster points, a contradiction.

The proof of the Lemma now breaks into three steps.

Step 1. By Lemma 2.4, we may choose an $N^{\prime} \subset N$ and real numbers $r$ and $\delta$ with $\delta>0$ so that for all $M \subset N^{\prime}$, there is an $x \in X$ with

$$
f_{m}(x)>r+\delta \text { for infinitely many } m \in M
$$

and

$$
f_{m}(x)<r \text { for infinitely many } m \in M \text {. }
$$

Step 2. For every $M \subset N^{\prime}$, let $K(M)$ equal the closure of the set of $x \in X$ satisfying (12); by Step $1, K(M)$ is nonempty. Then we may choose an $M \subset N^{\prime}$ so that $K\left(M^{\prime}\right)=K(M)$ for all $M^{\prime} \subset_{a} M$. Indeed if not, since $M^{\prime} \subset_{a} M$ implies $K\left(M^{\prime}\right) \subset K(M)$, we could choose by transfinite induction a family $\left(M_{\alpha}\right)_{\alpha<\omega_{1}}$, of (infinite) subsets of $N^{\prime}$ so that for all $\alpha<\beta<\omega_{1}$, $M_{\beta} \subset_{a} M_{\alpha}$ and $K\left(M_{\beta}\right) \subsetneq K\left(M_{\alpha}\right)$. The family $\left(K\left(M_{\alpha}\right)\right)_{\alpha<\omega}$, would thus be a strictly descending uncountable transfinite sequence of closed sets, and there exists no such family in a Polish space.

Step 3. Choose $M$ as in Step 2 and put $K=K(M)$. It follows that for every $M^{\prime} \subset M$ and non-empty relatively open subset $U$ of $K$, there exists $M^{\prime \prime} \subset M^{\prime}$ and $y, z$ in $U$ with

$$
\lim _{m \in M^{\prime \prime}} f_{m}(y) \geqslant r+\delta \text { and } \lim _{m \in M^{\prime \prime}} f_{n}(z) \leqslant r .
$$

Now let $U_{1}, U_{2}, \ldots$ be a base for the relatively open subsets of $K$. Then choose $M_{1} \supset M_{2} \supset \ldots$ subsets of $M$ and $y_{1}, z_{1}, y_{2}, z_{2}, \ldots$ so that for every $n, y_{n}, z_{n} \in U_{n}$ and (13) holds for $M^{\prime \prime}=M_{n}$ and $y=y_{n}, z=z_{n}$. Finally, choose $\mathbf{M}$ and $\mathbf{M} \subset_{a} M_{n}$ for all $n$, and set $L=\left\{y_{n}, z_{n}: n=1,2, \ldots\right\}$. It 
follows that $\left(f_{j}\right)_{j \in M}$ converges on $L$ to a function satisfying the Discontinuity Criterion.

REMARK. The above argument shows that if $\left(f_{n}\right)$ is any point-wise bounded sequence of real-valued functions on $X$ with no point-wise convergent subsequence, then there exists a subset $L$ of $X$ and a subsequence $\left(f_{n}^{\prime}\right)$ of $\left(f_{n}\right)$ converging on $L$ to a function $f$ satisfying the Discontinuity Criterion on $L$. It may be proved by a short direct argument that if $G$ is a uniformly bounded family of functions on $X$ so that such an $f$ is in the point-wise closure of $\{g \mid L: g \in G\}$, then $G$ contains a sequence $\left(g_{n}\right)$ satisfying the criterion of Proposition 2.3 (where " $S$ " of 2.3 equals $L$ ), and hence $\left(g_{n}\right)$ is equivalent to the usual $l^{1}$-basis. (See Proposition 2 of [52].) This gives a "topologicalanalytical" proof of Theorem 2.1 which by-passes the combinational Theorem 2.2 as well as the Baire Characterization Theorem; it also yields a proof of $1 \Rightarrow 5$ of Theorem 3 of $\$ 1$. See [52] for details.

The next result is far away from the final conclusion of 3.1; yet it seems to be central to the proof.

LeMma 3.9. Let $F$ be a relatively compact subset of $B_{1}(X)$. Then every element of the closure of $F$ lies in the closure of some countable subset of $F$.

We deduce this lemma from the following consequence of a special case:

Scноцium 3.10. Let $F$ be as in 3.9 so that $f \geqslant 0$ for all $f \in F$ and assume 0 is in the closure of $F$. Then for every $\varepsilon>0$ there exists a countable nonempty subset $H$ of $F$ with $\inf _{h \in H} h(x)<\varepsilon$ for all $x \in X$.

Proof. Suppose not. Then there is an $\varepsilon>0$ so that for every countable nonempty $H \subset F$,

$$
X(H) \text { is nonempty, where } X(H)=\left\{x \in X: \inf _{h \in H} h(x) \geqslant \varepsilon\right\} .
$$

Now if $H \subset H^{\prime}$ with $H^{\prime}$ a countable subset of $F$, then $X(H) \supset X\left(H^{\prime}\right)$. It follows that there exists a countable nonempty $H$ so that

$$
\overline{X(H)}=\overline{X\left(H^{\prime}\right)} \text { for all countable } H^{\prime} \subset F \text { with } H \subset H^{\prime} .
$$

Indeed, if not we choose by transfinite induction a family $\left(H_{\alpha}\right)_{\alpha<\omega_{1}}$ of countable subsets of $F$ so that for all $\alpha<\beta<\omega_{1}, H_{\alpha} \subset H_{\beta}$ and $\overline{X\left(H_{\alpha}\right)} \supsetneqq \overline{X\left(H_{\beta}\right)}$; the family $\left\{\overline{X\left(H_{\alpha}\right)}: \alpha<\omega_{1}\right\}$ is thus a strictly descending $\omega_{1}$-sequence of closed subsets of $X$, but there exists no such family in a Polish space.

Now choose $H$ as in (15) and let $D$ be a countable dense subset of $X(H)$. Since 0 is in the closure of $F$, there exists a sequence $f_{1}, f_{2}, \ldots$ in $F$ so that $f_{n}(d) \rightarrow 0$ for all $d \in D$. Finally set $K=\overline{X(H)}$ and let $g$ be a point-wise cluster point of the sequence $\left(f_{n}\right)$. Then $K$ is nonempty by (14) and $g$ vanishes on $D$, a dense subset of $K$. On the other hand, setting $H^{\prime}=H \cup\left\{f_{n}\right.$ : $n=1,2, \ldots\}$, then since $X\left(\left\{f_{n}: n=1,2, \ldots\right\}\right) \supset X\left(H^{\prime}\right), g(x)>\varepsilon$ for all $x \in X\left(H^{\prime}\right)$, but $X\left(H^{\prime}\right)$ is another dense subset of $K$ by (15). Hence $g \mid K$ has no points of continuity relative to $K$, so by 3.5 (the Baire-Characterization Theorem), $g \notin B_{1}(X)$, a contradiction.

Proof of Lemma 3.9. Let $F$ be as in 3.9, $g \in \bar{F}$, and $m$ be a positive 
integer. Let $X^{m}$ denote the $m$-fold product of $X$ with itself endowed with the product topology. Of course, $X^{m}$ is a Polish space. For any $h \in B_{1}(X)$, define $\phi_{m}(h)$ on $X^{m}$ by

$$
\phi_{m}(h)\left(x_{1}, \ldots, x_{m}\right)=\left|h\left(x_{1}\right)-g\left(x_{1}\right)\right|+\cdots+\left|h\left(x_{m}\right)-g\left(x_{m}\right)\right|
$$

for all $x_{1}, \ldots, x_{m}$ in $X$. Then $\phi_{m}(h) \in B_{1}\left(X^{m}\right)$ and $\phi_{m}: B_{1}(X) \rightarrow B_{1}\left(X^{m}\right)$ is a continuous map with $\phi_{m}(g)=0$. It follows that if $F_{m}=\phi_{m}(F)$, then $F_{m}$ is a relatively compact subset of $B_{1}\left(X^{m}\right)$ consisting of nonnegative functions with $0 \in \bar{F}_{m}$. Now it follows from Scholium 3.10 that there exists a countable nonempty set $H_{m} \subset F$ with $\inf _{h \in H_{m}} \phi_{m}(h)(y)<1 / m$ for all $y \in X^{m}$. Then setting $H=\cup_{m=1}^{\infty} H_{m}, H$ is a countable subset of $F$ with $g \in \bar{H}$. This completes the proof of Lemma 3.9.

The preceding results and arguments are due to the author [54]. The remaining steps in the proof are due to Bourgain, Fremlin and Talagrand [7]. The heart of the entire matter is contained in the following lemma, which we refer to later as the main step:

LEMMA 3.11. Let $\left(f_{n}\right)$ be a sequence of continuous functions on $X$ so that 0 is a point-wise cluster point of $\left(f_{n}\right)$. Assume that every subsequence of $\left(f_{j}\right)$ has a further point-wise convergent subsequence. Then some subsequence of $\left(f_{j}\right)$ converges point-wise to zero.

Let us say that an (infinite) $M \subset N$ is good provided 0 is a point-wise cluster point of $\left\{f_{n}: n \in M\right\}$. The proof of 3.11 requires three preliminary results about good sets, the first of which requires all of the machinery already developed. We first state these three results, then deduce (3.11) from them, and finally prove the three results.

Scholium 3.12. Given good sets $M_{1}, M_{2}, \ldots$ with $M_{1} \supset_{a} M_{2}$ $\supset_{a} \ldots \supset_{a} M_{n} \supset_{a} M_{n+1}, \ldots$, there exists a good set $M$ with $M_{n} \supset_{a} M$ for all $n$.

Now fix $\varepsilon>0$ and for any $M \subset N$, let $K_{M}$ denote the closure of $\{x \in X$ : $\left.\varlimsup_{m \in M} f_{m}(x)>\varepsilon\right\}$.

Scholium 3.13. For any good set $N^{\prime}$ there exists a good set $M$ with $M \subset N^{\prime}$ so that $K_{M}=K_{L}$ for any good $L \subset N^{\prime}$ with $L \subset_{a} M$.

Scholium 3.14. Let $M$ be as in 3.13. Then $K_{M}$ is empty. That is, $\varlimsup_{m \in M}\left|f_{m}(x)\right|<\varepsilon$ for all $x \in X$.

3.11 now follows quickly. Indeed the preceding two Scholia show that for every good $N^{\prime}$ and any $\varepsilon>0$, there exists a good $M \subset N^{\prime}$ with $\varlimsup_{m \in M}\left|f_{m}(x)\right| \leqslant \varepsilon$ for all $x \in X$. It follows that we may choose good sets $M_{1}, M_{2}, \ldots$ so that for all $n, M_{n} \supset M_{n+1}$ and $\overline{\lim }_{m \in M_{n}}\left|f_{m}(x)\right|<1 / n$ for all $x \in X$. Now choose integers $m_{1}<m_{2}<\ldots$ with $m_{n} \in M_{n}$ for all $n$. Then $f_{m} \rightarrow 0$ point-wise.

We pass now to the proof of the three Scholia. We first note the following immediate stability properties of good 
If $M$ is good and $M=L_{1} \cup L_{2}$, then either $L_{1}$ or $L_{2}$ is good. If $M$ is good, $\varepsilon>0$, and $G$ is a finite subset of $X$, then $\left\{m \in M:\left|f_{m}(x)\right|<\varepsilon\right.$ for all $\left.x \in G\right\}$ is good.

Next, we note that any subsequence of $\left(f_{n}\right)$ has a Baire-1 cluster point. Indeed, the subsequence has a further point-wise convergent subsequence by hypothesis; the limit function is Baire-1 since the $f_{n}$ 's are continuous. Thus by Lemma 3.7, $\left\{f_{1}, f_{2}, \ldots\right\}$ is a relatively compact subset of $B_{1}(X)$.

Proof of SCHOLIUM 3.12. Let $F$ denote the set of all limits of point-wise convergent sequences $\left(f_{j}\right)_{j \in M}$ with $M \subset_{a} M_{n}$ for all $n$. Since $F$ is contained in the point-wise closure of $\left\{f_{1}, f_{2}, \ldots\right\}, F$ is a relatively compact subset of $B_{1}(X)$. We first observe that $0 \in \bar{F}$. Indeed, let $\varepsilon>0$ and let $G$ be a finite subset of $X$. We need to show that there is an $f \in F$ so that

$$
|f(x)|<\varepsilon \text { for all } x \in G .
$$

Since the $M_{j}$ 's are good, we may choose $m_{1}, m_{2}, \ldots$ so that for all $j$, $m_{j}<m_{j+1}, m_{j} \in M_{j}$, and $\left|f_{m_{j}}(x)\right|<\varepsilon / 2$ for all $x \in G$. Now let $M$ be an (infinite) subset of $\left\{m_{1}, m_{2}, \ldots\right\}$ so that $\left(f_{m}\right)_{m \in M}$ converges point-wise to a function $f$. Then $M \subset_{a} M_{j}$ for all $j$ so $f \in F$ and $|f(x)| \leqslant \varepsilon / 2$ for all $x \in G$; thus $f$ satisfies (18).

Now by Lemma 3.9, 0 is in the closure of some countable subset $\left\{g_{1}, g_{2}, \ldots\right\}$ of $F$. For each $j$, we may choose an $N_{j}$ so that $\left(f_{n}\right)_{n \in N_{j}}$ converges point-wise to $g_{j}$ and

$$
N_{j} \underset{a}{\subset} M_{n} \text { for all } n
$$

We now assert that

$$
\text { there exists an } M \text { with } N_{j} \underset{a}{\subset} M \underset{a}{\subset} M_{n} \text { for all } j \text { and } n \text {. }
$$

Once (20) is established, we have that $M$ is the desired good set. Indeed, $g_{j} \in \overline{\left\{f_{m}: m \in M\right\}}$ for all $j$, hence $0 \in \overline{\left\{g_{1}, g_{2}, \ldots\right\}} \subset \overline{\left\{f_{m}: m \in M\right\}}$.

(20) in turn is a classically known consequence of the fact that (19) holds for all $j$. Here is a quick proof: For each $j$, set $A_{j}=\cup_{i=1}^{j} N_{i}$ and $B_{j}=$ $\bigcap_{i=1}^{j} M_{i}$. Then also $A_{j} \subset_{a} B_{n}$ for all $j$ and $n$. Now choose $n_{1}<n_{2}<\ldots$ so that for all $j, A_{j} \cap\left(n_{j}, \infty\right) \subset B_{j}$. Then $M=\cup_{j=1}^{\infty} A_{j} \cap\left(n_{j}, n_{j+1}\right]$ serves as the desired set.

REMARK. The above argument may be "abstracted" to yield the following result: Let $Y$ be a regular sequentially compact Hausdorff space so that for any subset $F$ of $Y$, any point in the closure of $F$ is in the closure of some countable subset of $F$. Then given sequences $Y_{1}, Y_{2}, \ldots$ in $Y$ and $y \in Y$ so that for all $j, Y_{j+1}$ is a subsequence of $Y_{j}$ and $y$ is a cluster point of $Y_{j}$, there exists a sequence $Z$ in $Y$ which is a subsequence of all the $Y_{j}^{\prime}$ 's so that $y$ is a cluster point of $Z$.

Scholium 3.13 follows easily from 3.12 by transfinite induction. Indeed, if $M \supset_{a} M^{\prime}$ then $K_{M} \supset K_{M}^{\prime}$. Thus were 3.13 false, using 3.12 and (9) we could choose a family $\left(M_{\alpha}\right)_{\alpha<\omega_{1}}$ of good subsets of $N^{\prime}$ so that for all $\alpha<\beta<\omega_{1}$, $M_{\alpha} \supset_{a} M_{\beta}$ and $K_{M_{\alpha}} \supsetneq K_{M_{\beta}}$, which is impossible since $X$ is Polish.

Proof of Scholium 3.14. Let $M$ be as in 3.13 and suppose $K=K_{M}$ is nonempty. We shall exhibit a point-wise cluster point $f$ of $\left(f_{m}\right)_{m \in M}$ and dense 
subsets $A$ and $B$ of $K$ so that $f$ vanishes on $A$ and $f$ is at least $\varepsilon$ on $B$, i.e. $|f(b)|>\varepsilon$ for all $b \in B$. Then $f \mid K$ has no points of continuity relative to $K$, hence $f \notin B_{1}(X)$ contradicting the fact that $\left\{f_{1}, f_{2}, \ldots\right\}$ is a relatively compact subset of $B_{1}(X)$.

We may take for $A$ a countable dense subset $\left\{a_{1}, a_{2}, \ldots\right\}$ of $K$. It follows from (17) that we may choose good sets $L_{1}, L_{2}, \ldots$ so that for all $n$ and $1<i<n, M \supset L_{n} \supset L_{n+1}$ and $\left|f_{j}\left(a_{i}\right)\right|<1 / n$ for all $j \in L_{n}$. Now by the crucial Scholium 3.12, choose a good $L$ with $L \subset_{a} L_{n}$ for all $n$. It follows that any point-wise cluster point of $\left(f_{l}\right)_{l \in L}$ vanishes on $A$ since $\left(f_{l}\right)_{l \in L}$ tends point-wise to zero on $A$; moreover by the definition of $M, K_{l}=K$. The discovery of the set $B$ is more delicate.

To simplify notation, we now let $L=N$ itself (which we could certainly do by just renumbering $\left.\left(f_{l}\right)_{l \in L}\right)$. Thus for any good $R, K_{R}=K$ and $f_{n} \rightarrow 0$ point-wise on $A$. We shall also just work in the topological space $K$, which of course is a Polish space, being a closed subset of one. (Thus, "open subset of $K$ " refers to a subset of $K$ open in its relative topology.) We note next that if $U$ is a nonempty open subset of $K$ and $M^{\prime}$ is a good set, then $L_{1}=\left\{l \in M^{\prime}\right.$ : $\left|f_{l}(u)\right|>\varepsilon$ for some $\left.u \in U\right\}$ is a good set. If not, then by (16), $L_{2}=M^{\prime} \sim L_{1}$ is good. However then $K_{L_{2}} \cap U=\varnothing$, which implies $K_{l_{2}} \neq K$, contradicting the definition of $K$. It follows immediately by induction that

for any finite number $U_{1}, \ldots, U_{k}$ of nonempty open subsets of $K, G$ is good (and hence infinite), where $G=\{n \in N$ : for all $1 \leqslant i \leqslant k$ there is $a u \in U_{i}$ with $\left.\left|f_{n}(u)\right|>\varepsilon\right\}$.

At last we shall use in an essential way the continuity of the $f_{n}$ 's and the completeness of $K$ under some appropriate metric. Choose a complete metric inducing $K$ 's topology and let $B_{1}, B_{2}, \ldots$ be a base for the nonempty open subsets of $K$. We shall exhibit a sequence $m_{1}<m_{2}<\ldots$ of integers so that for each $i$, there exists a $b_{i} \in B_{i}$ with $\lim \left|f_{m_{j}}\left(b_{i}\right)\right| \geqslant \varepsilon$. Then we set $B=\left\{b_{i}\right.$ : $i=1,2, \ldots\}$; any cluster point $f$ of $\left(\overline{f_{m}}\right)$ produces the desired contradiction.

The idea of the construction of the $m_{i}$ 's and $b_{i}$ 's is quite simple. For example, to produce $b_{1}$ alone, we just use the " $k=1$ " version of (21) along with the continuity of the $f_{j}$ 's to produce $l_{1}<l_{2}<\ldots$ and nonempty closed neighborhoods $U_{1} \supset U_{2} \supset \ldots$ with diameters going to zero, $U_{1} \subset B_{1}$, and $f_{l}>\varepsilon$ on $U_{j}$ for all $j$. By completeness there exists a point $b_{1}$ belonging to all the $U_{j}$ 's and then $\lim \left|f_{l_{j}}\left(b_{1}\right)\right| \geqslant \varepsilon$. Now we just carry out this procedure simultaneously for all the $B_{j}$ 's. Thus, we construct open sets $U_{i}^{j}$ and integers $m_{j}$ having the following properties for all $i$ with $1 \leqslant i<\infty$ and $j$ with $i<j<\infty$ :

(a) $U_{i}^{i-1}=B_{i}$,

(b) $U_{i}^{j}$ is nonempty with $\bar{U}_{i}^{j} \subset U_{i}^{j-1}$,

(c) the diameter of $U_{i}^{j}$ is at most $1 / j$, and

(d) $\left|f_{m_{j}}(u)\right|>\varepsilon$ for all $u \in U_{i}^{j}$ and $m_{j}>m_{j-1}$ (where $m_{0}=0$ ).

Once this is done, then fixing $i$, by completeness and (b) and (c) we may choose a (unique) $b_{i} \in \bigcap_{j=i}^{\infty} \vec{U}_{i}^{j}=\bigcap_{j=i}^{\infty} U_{i}^{j}$. By (a) and (b), $b_{i} \in B_{i}$ and $\left|f_{m_{i}}\left(b_{i}\right)\right|>\varepsilon$ for all $j \geqslant i$ by (d) thus completing the proof.

The construction by induction goes as follows: Define $U_{i}^{i-1}=B_{i}$ for all $i$. 
By (21) there is an $m_{1}$ and a $u \in B_{1}$ with $\left|f_{m_{1}}(u)\right|>\varepsilon$. By continuity of $f_{m_{1}}$ we choose an open $U_{1}^{1}$ containing $u$ with diameter at most equal to $1, \bar{U}_{1}^{1} \subset B_{1}$, and $\left|f_{m_{1}}(v)\right|>\varepsilon$ for all $v \in U_{1}^{1}$. Suppose then $m_{n}$ and open nonempty sets $U_{1}^{n}, \ldots, U_{n}^{n}$ have been chosen. By (21), the set $G=\{j \in N$ : for $1 \leqslant i \leqslant n$ +1 , there is a $u \in U_{i}^{n}$ with $\left.\left|f_{j}(u)\right|>\varepsilon\right\}$ is infinite. Hence we may choose $m_{n+1}>m_{n}$ with $m_{n+1} \in G$. Now for each $i$ with $1 \leqslant i \leqslant n+1$, choose by continuity of $f_{m_{n+1}}$ a nonempty open set $U_{i}^{n+1}$ of diameter at most $1 /(n+1)$ with $\bar{U}_{i}^{n+1} \subset U_{i}^{n+1}$ and $\left|f_{m_{n+1}}(u)\right|>\varepsilon$ for all $u \in U_{i}^{n+1}$. This completes the construction of the $f_{m}$ 's and $U_{i}^{j}$ 's, and thus the proof of our main step, Lemma 3.11, is complete. In order to complete the proof of Theorem 3.1, we need one final deep classical fact about Polish spaces. (This result is at least 50 years old; for a proof, see [36].)

Lemma 3.15. Let $X$ and $Y$ be Polish spaces and $\phi: X \rightarrow Y$ a Borel measurable map; set $E=\phi(X)$. Then there exists a Polish space $Z$ and a continuous surjection $s: Z \rightarrow E$.

(It is known that $Z$ may be chosen to be the "standard" Polish space $N^{N}$. 3.15 may be rephrased as stating that analytic sets are closed under Hausdorff Borel images, where an analytic set is defined as a continuous Hausdorff image of some Polish space.)

CoMPLETION OF THE PROOF OF THEOREM 3.1. Let $F$ be a relatively compact subset of $B_{1}(X)$ and let $f$ belong to the closure of $F$. Since $B_{1}(X)$ is a linear topological space, $F-f=\{g-f: g \in F\}$ is also a relatively compact subset of $B_{1}(X)$ and 0 belongs to the closure of $F-f$. Once we show there is a sequence in $F-f$ converging point-wise to zero, we obtain that $f$ is in the sequential closure of $F$. Hence we may and shall assume that $f=0$. Now by Lemma 3.9 there exists a sequence $f_{1}, f_{2}, \ldots$ in $F$ so that 0 is a cluster point of $\left(f_{j}\right)$. By Lemma $3.8 F$ is sequentially compact and hence every subsequence of $\left(f_{j}\right)$ has a further point-wise convergent subsequence. If the $f_{j}$ 's were all continuous, the Main Step 3.11 would complete the proof. But Lemma 3.15 implies that countably many real-valued Borel measurable functions defined on a Polish space are all continuous under some finer Polish topology on the space. We prefer to go through a direct proof of the part of this (known) implication that we need. Let $Y$ denote the Polish space $X \times \mathbf{R}^{\infty}$ and denote its elements by sequences $\left(x, r_{1}, r_{2}, \ldots\right)$. Since the $f_{j}^{\prime}$ 's are Baire-1 they are Borel measurable. It is readily verified that $\phi: X \rightarrow Y$ defined by $\phi(x)=$ $\left(x, f_{1}(x), f_{2}(x), \ldots\right)$ for all $x \in X$, is a Borel measurable map. Let $E=\phi(X)$ and choose by Lemma 3.15 a Polish space $Z$ and a surjection $s: Z \rightarrow E$. For each $n$, define $\tilde{f}_{n}: Z \rightarrow \mathbf{R}$ by $\tilde{f}_{n}(z)=(s(z))_{n+1}$. Then $\left(\tilde{f}_{n}\right)$ is a sequence of continuous functions on $Z$. It is easily verified that a subsequence of $\left(\tilde{f}_{n}\right)$ converges point-wise on $Z$ if and only if the corresponding subsequence of $f_{n}$ 's converges point-wise on $X$, and moreover 0 is in the point-wise closure of $\left(\tilde{f}_{n}\right)$. It follows that $\left(\tilde{f}_{n}\right)$ satisfies the hypotheses of Lemma 3.11 on the Polish space $Z$ and hence there is a sequence $n_{1}<n_{2}<\ldots$ with $\tilde{f}_{n_{j}} \rightarrow 0$ point-wise. Then $f_{n_{j}} \rightarrow 0$ point-wise completing the proof.

We conclude with a brief discussion of some generalizations of Theorem 3.1 also due to Bourgain, Fremlin and Talagrand [7]. One says that a regular Hausdorff space $Y$ is angelic (as in [47]) if any subset $F$ of $Y$ such that every 
countable subset of $F$ has a cluster point in $Y$ is relatively compact and sequentially dense in its closure. Thus Theorem 3.1 asserts precisely that $B_{1}(X)$ is angelic (where $X$ as always denotes a fixed Polish space). Now let $B(X)$ denote the space of all Borel measurable real-valued functions defined on $X$, endowed with the topology of point-wise convergence. Then it is proved in [7] that countable relatively countably compact subsets of $B(X)$ have compact, angelic closed convex hulls. In particular, this implies that compact subsets of $B(X)$ are sequentially compact and countable relatively compact subsets are sequentially dense in their closures, thus answering several questions raised in [54]. It also implies that if $A$ is a subset of the continuous functions on $X$ such that every sequence in $A$ has a Borel measurable cluster point, then $A$ is a relatively compact subset of $B_{1}(X)$. For then $A$ is sequentially compact in $B(X)$, and any limit of a convergent sequence of elements of $A$ is Baire-1. The last fact, however, is in reality the base for the more general results. Using generalizations of our Lemma 3.7 and combinational Theorem 2.2, it is shown in [7] that if $\left(f_{n}\right)$ is a point-wise bounded sequence in $C(X)$ with no point-wise convergent subsequence, then $\left(f_{n}\right)$ has a subsequence $\left(f_{n}^{\prime}\right)$ with no Borel-measurable cluster points. (We give a somewhat different proof of this below.) Assuming only this result, suppose that $F$ is a relatively countably compact separable subset of $B(X)$ and let $f_{1}, f_{2}, \ldots$ be a sequence in $F$ with $\bar{F}=\left\{f_{1}, f_{2}, \ldots\right\}$. Then there exists a finer Polish topology $\Upsilon$ on $X$ so that $f_{1}, f_{2}, \ldots$ are all continuous on $(X, \Upsilon)$ (see our remarks in the proof of Theorem 3.1 following 3.15 above). But then every subsequence of $\left(f_{n}\right)$ has a further point-wise convergent subsequence. Indeed, if not, there would exist a subsequence $\left(f_{n}^{\prime}\right)$ with no cluster points in $B(X, \Upsilon)$. But by hypothesis there is a $g \in B(X)$ which is a cluster point of $\left(f_{n}^{\prime}\right)$; then $g \in B(X, \Upsilon)$ also since $\Upsilon$ is a finer topology on $X$. Thus by Theorem $3.1\left\{f_{j}: j=1,2, \ldots\right\}$ is a relatively compact subset of $B_{1}(X, \Upsilon)$ which is moreover sequentially dense in its point-wise closure $H$. But then $H$ is a compact subset of $B(X)$ since a point-wise limit of a sequence of Borel measurable functions is again Borel measurable, and, of course, $H$ equals the sequential closure of $F$. Actually, we have seen that a separable compact subset of $B(X)$ is angelic simply because it is homeomorphic to a compact subset of $B_{1}(Y)$ for some Polish space $Y$. It is apparently unknown if either of these assertions are true of arbitrary compact subsets of $B(X)$. The authors of [7] also obtain further generalizations of Theorem 3.1 to more general classes of topological spaces $X$ as well as more general classes of measurable functions. Most of these generalizations boil down to an elaboration of the following concrete fact established there: If a point-wise bounded sequence $\left(f_{n}\right)$ in $C([0,1])$ has no point-wise convergent subsequence, then it has a subsequence $\left(f_{n}^{\prime}\right)$ so that every point-wise cluster point of $\left(f_{n}^{\prime}\right)$ is nonmeasurable in every conceivable sense.

Here is a proof of the existence of $\left(f_{n}^{\prime}\right)$, following the lines of argument of [7] but differing somewhat in detail. We first consider the simplest possible case, working on the Cantor set rather than $[0,1]$.

LEMma 3.16. Let $\Delta$ equal $\{0,1\}^{N}$, the set of all infinite sequences of 0 's and 1 's endowed with the product topology and let $m$ denote the natural probability measure on the Borel subsets of $\Delta$. Let $\varepsilon_{n}(x)=x(n)$ for all $x \in \Delta$ and $n \in N$, 
and let $h$ be a point-wise cluster point of $\left(\varepsilon_{n}\right)$. Then $h$ is not measurable with respect to the completion of $m$.

Proof. $\Delta$ is a compact abelian group under the operation $(x+y)(n)=$ $x(n)+y(n) \bmod 2$ for all $x, y \in F$ and $n \in N$, and $m$ is the (unique) normalized Haar measure on $G$.

Let us say that a subset of $\Delta$ is $m$-measurable if it is measurable with respect to the completion of $m$. Then it is a classical fact that if $E$ is an $m$-measurable subset of positive measure, then $E+E$ contains a nonempty open set, where $E+E=\{e+f: e \in E$ and $f \in E\}$. The simplest conceptual proof is to use convolution. Set $\chi_{E} * \chi_{E}(y)=\int_{\Delta} \chi_{E}(y+$ $x) \chi_{E}(x) d m(x)$. Then $\chi_{E} * \chi_{E}$ is continuous and supported on $E+E$, and of course $\left(\chi_{E} * \chi_{E}\right)(0)=m(E)$.

For $x \in \Delta$, set $\tilde{x}=1-x$, and for $E \subset \Delta$, set $\tilde{E}=\{\tilde{x}: x \in \Delta\}$. Then it is also well known that if $E$ is $m$-measurable, so is $\tilde{E}$ and $m(E)=m(\tilde{E})$.

Now let $h$ be a point-wise cluster point of $\left(\varepsilon_{n}\right)$ and let $E=\{x: h(x)=1\}$. We then have by the definition of the topology of point-wise convergence that

for any $k$ and $x_{1}, \ldots, x_{k}$ in $G$, there are infinitely many $n \in N$ with $h\left(x_{i}\right)=x_{i}(n)$ for all $i=1,2, \ldots, k$.

We then easily obtain

(i) $E$ is dense in $\Delta$,

(ii) $\tilde{E}=\sim E$,

and (iii) $E+E$ is disjoint from $E$.

Indeed, applying (22) for $k=1$, we obtain that $x \in E$ if $x(n)=1$ for all $n$ sufficiently large, and of course the set of such $x$ is dense. Let $x \in \Delta$. Then applying (22) for $k=2, x_{1}=x, x_{2}=\tilde{x}$, we obtain that there is an $n \in N$ with $h(x)=x(n)$ and $h(\tilde{x})=\tilde{x}(n)=1-x(n)$. Thus either $x$ or $\tilde{x}$ belongs to $E$ while if $x \in E, h(\tilde{x})=0$ so $\tilde{x} \notin E$, proving (ii). Finally, let $x, y \in E$. Applying (22) for $k=3$, we may choose an $n \in N$ with $h(x)=x(n), h(y)=$ $y(n)$ and $h(x+y)=(x+y)(n)$. Since $x, y \in E, x(n)=y(n)=1$, hence $(x+y)(n)=0$, so $x+y \notin E$.

It now follows immediately that $E$ is nonmeasurable. Indeed, suppose not. Then also $\tilde{E}$ would be $m$-measurable, so by 23 (ii), $m(E)+m(\tilde{E})=2 m(E)$ $=1$, or $m(E)=\frac{1}{2}$. Thus $E$ would be of positive measure, hence $E+E$ would contain a nonempty open set. But then $E$ would not be dense in $\Delta$ by 23 (iii), contradicting 23 (i).

RemarKs. Define $\phi: \Delta \rightarrow[0,1]$ by $\phi(x)=\sum_{j=1}^{\infty} x(j) / 2^{j}$ for all $x \in \Delta$. It is easily seen that $\phi(E)$ is not Lebesgue-measurable, where $E$ is as above. This is due to W. Sierpinski (see Fonctions additive non complètement additives et fonctions non-mesurables, Fund. Math. 30 (1938), 96-99). In fact, he obtains essentially that $\phi(E)$ is of inner measure zero and outer measure one. In our context, to see this it suffices to show that any $m$-measurable subset of $E$ is of measure zero, for then thanks to the fact that $x \rightarrow \tilde{x}$ is a measure preserving transformation, any measurable subset of $\tilde{E}=\sim E$ is also of measure zero; 
and of course the outer measure of $E$ equals the inner measure of $\tilde{E}$. Now setting $D=\{x \in \Delta: x(n)=0$ for all $n$ sufficiently large $\}$, then $D$ is a subgroup of $\Delta$ and (22) implies that $x \in E$ if and only if $x+d \in E$ for all $d \in D$; i.e. $E+D=E$. Now if $F$ were a subset of $E$ of positive measure, then (23) (ii) implies that $0<m(F) \leqslant \frac{1}{2}$, since $m(F)=m(\tilde{F})$ and $F \cap \tilde{F}=$ $\varnothing$. But then also $F+D$ is a measurable subset of $E$, hence $0<m(F+D) \leqslant \frac{1}{2}$. However, it is a classical fact that since $(F+D)+D$ $=F+D, m(F+D)=0$ or $m(F+D)=1$. (This is essentially Sierpiński's proof that $\phi(E)$ is nonmeasurable.) Now it is also easily seen using (22) that Q $=\{H \subset N$ : there is an $x \in E$ with $H=\{n: x(n)=1\}\}$ is a nonprincipal ultrafilter, and conversely any nonprincipal ultrafilter gives rise to a cluster point $h$ of $\left(\varepsilon_{n}\right)$. Thus the above lemma is equivalent to the assertion that any nonprincipal ultrafilter in $N$ is nonmeasurable, as proved in Lemma 2D of [7].

Now let $\left(\varepsilon_{n}\right)$ be as in 3.16 and let $U_{n}=\left\{x: \varepsilon_{n}(x)=0\right\}$ for all $n$. Of course $\left(U_{n}\right)$ is a Boolean and statistically-independent sequence of sets. The next result, similar to Lemma 2D of [7], shows that any sequence of closed pairs with no convergent subsequence contains a subsequence which looks exactly like $\left(U_{n}, \sim U_{n}\right)$ relative to some compact set.

THEOREM 3.17. Let $X$ be a Polish space and $\left(A_{n}, B_{n}\right)_{n \in N}$ a sequence of pairs of subsets of $X$ with $A_{n}, B_{n}$ closed and $A_{n} \cap B_{n}=\varnothing$ for all $n$. Assume that $\left(A_{n}, B_{n}\right)_{n \in N}$ has no convergent subsequence. Then there exists a compact subset $K$ of $X$ homeomorphic to $\Delta$, a homeomorphism $\sigma$ from $K$ onto $\Delta$, and a sequence $n_{1}<n_{2}<\ldots$ so that $A_{n_{j}} \cap K=\sigma^{-1}\left(U_{j}\right)$ and $B_{n_{j}} \cap K=\sigma^{-1}\left(\sim U_{j}\right)$ for all $j$.

Proof. Endow $X$ with a complete metric inducing its topology. We first observe that if $\tilde{X}$ is a closed subset of $X$ and $M \subset N$ is such that $\left(A_{n}, B_{n}\right)_{n \in M}$ has no subsequence convergent on $\tilde{X}$, then for any $\varepsilon>0$ there exists an $L \subset M$ so that $\left(A_{n}, B_{n}\right)_{n \in L}$ has no subsequence convergent on a fixed closed subset of $\tilde{X}$ of diameter less than $\varepsilon$. Indeed, if not, choose closed sets $D_{1}, D_{2}, \ldots$ of diameter less than $\varepsilon$ with $\tilde{X}=\cup_{j=1}^{\infty} D_{j}$. Then choose $M_{1} \supset$ $M_{2} \supset \ldots$ with $M_{1} \subset M$ so that $\left(A_{n}, B_{n}\right)_{n \in M_{j}}$ converges on $D_{j}$. Then choosing $L$ with $L \subset_{a} M_{j}$ for all $j,\left(A_{n}, B_{n}\right)_{n \in L}$ converges on $\tilde{X}$, a contradiction.

The construction of $K$ and $\sigma$ now follows easily from Lemma 2.6, as in our proof of Theorem 2.2. First choose $X_{\phi}$ a closed subset of $X$ of diameter less than 1 and $N^{\prime} \subset N$ so that $\left(A_{j}, B_{j}\right)_{j \in N^{\prime}}$ has no subsequence convergent on $X_{\phi}$. Then by Lemma 2.6, choose $n_{1} \in N^{\prime}$ and $N_{1} \subset N^{\prime}$ with $n>n_{1}$ for all $n \in N_{1}$ so that $\left(A_{j}, B_{j}\right)_{j \in N_{1}}$ has no subsequence convergent on $A_{n_{1}} \cap X_{\phi}$ or $B_{n_{1}} \cap X_{\phi}$. Set $X_{0}=A_{n_{1}} \cap X_{\phi}$ and $X_{1}=B_{n_{1}} \cap X_{\phi}$. Suppose $k>1, n_{k}, N_{k} \subset$ $N$, and closed sets $X_{\varepsilon_{1}} \ldots, \varepsilon_{k}$ have been constructed for all $\left(\varepsilon_{1}, \ldots, \varepsilon_{k}\right)$ with $\varepsilon_{i}=0$ or 1 so that $X_{\varepsilon_{1}, \ldots, \varepsilon_{k}} \cap X_{\varepsilon_{1}^{\prime}, \ldots, \varepsilon_{k}^{\prime}}=\varnothing$ if $\left(\varepsilon_{1}, \ldots, \varepsilon_{k}\right) \neq\left(\varepsilon_{1}^{\prime}, \ldots, \varepsilon_{k}^{\prime}\right)$ and $\left(A_{j}, B_{j}\right)_{j \in N_{k}}$ has no subsequence convergent on $X_{\varepsilon_{1}, \ldots, \varepsilon_{k}}$ for all $\left(\varepsilon_{1}, \ldots, \varepsilon_{k}\right)$. By applying our initial observation $2^{k}$ times, we may choose $N_{k}^{\prime} \subset N_{k}$ and closed sets $\tilde{X}_{\varepsilon_{1}}, \ldots, \varepsilon_{k}$ so that for all $\left(\varepsilon_{1}, \ldots, \varepsilon_{k}\right), \tilde{X}_{\varepsilon_{1}}, \ldots, \varepsilon_{k} \subset$ $X_{\varepsilon_{1}, \ldots, \varepsilon_{k}}, \tilde{X}_{\varepsilon_{1}, \ldots \varepsilon_{k}}$ has diameter less than $\frac{1}{k},\left(A_{j}, B_{j}\right)_{j \in N_{k}^{\prime}}$ has no subsequence convergent on $X_{\varepsilon_{1}}, \ldots, \varepsilon_{k}$, and $n>n_{k}$ for all $n \in N_{k}^{\prime}$. We now apply Lemma 2.6 to choose $n_{k+1} \in N_{k}^{\prime}$ and $N_{k+1} \subset N_{k}^{\prime}$ so that for all $\left(\varepsilon_{1}, \ldots, \varepsilon_{k}\right)$, $\left(A_{n}, B_{n}\right)_{n \in N_{k+1}}$ has no subsequence convergent on $A_{n_{k+1}} \cap \tilde{X}_{\varepsilon_{1}}, \ldots, \varepsilon_{k}$ or $B_{n_{k+1}} \cap$ 
$\tilde{X}_{\varepsilon_{1}, \ldots, \varepsilon_{k}} \cdot$ We then set $X_{\varepsilon_{1}, \ldots, \varepsilon_{k}, 0}=A_{n_{k+1}} \cap \tilde{X}_{\varepsilon_{1}, \ldots, \varepsilon_{k}}$ and $X_{\varepsilon_{1}, \ldots, \varepsilon_{k}, 1}=B_{n_{k+1}} \cap$ $\tilde{X}_{\varepsilon_{1}, \ldots, \varepsilon_{k}}$ for all $\varepsilon_{1}, \ldots, \varepsilon_{k}$.

We now simply set $K=\bigcap_{k=1}^{\infty} \cup X_{\varepsilon_{1}}, \ldots, \varepsilon_{k}$, where the union is taken over all $\left(\varepsilon_{1}, \ldots, \varepsilon_{k}\right)$ with $\varepsilon_{i}=0$ or 1 for all $i$.

Define $\sigma: K \rightarrow \Delta$ by $\sigma(k)=x$ provided $k \in \bigcap_{k=1}^{\infty} X_{x(1), \ldots, x(k)}$. Since $X$ is a complete metric space, it follows that $\sigma$ is a surjective homeomorphism satisfying the conclusion of 3.17 .

We now easily obtain the following result of [7]:

TheOREM 3.18. Let $X$ be a Polish space and $\left(f_{n}\right)$ a point-wise bounded sequence of continuous functions on $X$ with no point-wise convergent subsequence. Then there exists a subsequence $\left(f_{n}^{\prime}\right)$ of $\left(f_{n}\right)$, a compact nonempty subset $K$ of $X$, and a probability measure $\mu$ on the Borel subsets of $K$ so that no point-wise cluster point of $\left(f_{n}^{\prime}\right)$ is measurable with respect to the completion of $\mu$. (Thus no cluster point of $\left(f_{n}^{\prime}\right)$ is Borel-measurable or, in fact, universally measurable.)

Proof. By Lemma 2.4, there exists an $N^{\prime} \subset N$ and real numbers $r$ and $\delta$ with $\delta>0$ so that $\left(\tilde{A}_{n}, \tilde{B}_{n}\right)_{n \in N^{\prime}}$ has no convergent subsequence, where $\tilde{B}_{n}=\left\{x \in X: f_{n}(x)>r+\delta\right\}$ and $\tilde{A}_{n}=\left\{x \in X: f_{n}(x)<r\right\}$. But then if $B_{n}=\left\{x \in X: f_{n}(x) \geqslant r+\delta\right\}, A_{n}=\left\{x \in X: f_{n}(x) \leqslant r\right\},\left(A_{n}, B_{n}\right)_{n \in N^{\prime}}$ has no convergent subsequence either. Since the $f_{n}$ 's are continuous, $A_{n}$ and $B_{n}$ are closed for all $n$. Now choose $K \subset X, n_{1}<n_{2}<\ldots$ and a homeomorphism $\sigma$ from $K$ onto $\Delta$ satisfying the conclusion of Theorem 3.17. Finally, let $m$ be as in Lemma 3.16 and let $\mu$ be the Borel measure on $K$ defined by $\mu(B)=m(\sigma(B))$ for all Borel $B \subset K$. Now let $g$ be a point-wise cluster point of $\left(f_{n}\right)_{j=1}^{\infty}$. Then we claim $g \mid K$ is not measurable with respect to the completion of $\mu$. Indeed, if it were and if $\tilde{h}(x)=1$ if $g(x) \geqslant r+\delta$, $\tilde{h}(x)=0$ if $g(x) \leqslant r$, then $\tilde{h}$ would be $\mu$-measurable and a point-wise cluster pcint of $\left(\chi_{B_{n}}\right)_{j=1}^{\infty}$. It follows that $h=\tilde{h} \circ \sigma$ would be $m$-measurable and a point-wise cluster point of $\left(\varepsilon_{j}\right)$, contradicting Lemma 3.16.

\section{REFERENCES}

1. D. E. Alspach, Quotients of $C[0,1]$ with separable dual, Bull. Amer. Math. Soc. 83 (1977), 1057-1059.

2. D. Amir, Projections onto continuous function spaces, Proc. Amer. Math. Soc. 15 (1964), $396-402$.

3. R. Baire, Sur les fonctions des variables réelles, Ann. Mat. Pura Appl. (3) 3 (1899), 16, 30.

4. S. Banach, Théorie des operations linéaires, Warszawa, 1932; Reprint, Chelsea, New York.

5. Y. Benyamini, An extension theorem for separable Banach spaces (to appear).

6. C. Bessaga and A. Pełczyński, A generalization of results of $R$. C. James concerning absolute bases in Banach spaces, Studia Math. 17 (1958), 166-174.

7. J. Bourgain, D. H. Fremlin and M. Talagrand, Pointwise compact sets of Baire measurable functions, Amer. J. Math. (to appear).

8. A. Brunel and L. Sucheston, Sur les amarts faibles à valeurs vectorielles, C. R. Acad. Sci. Paris Sér. A 283 (1976), 1011-1014.

9. G. Choquet, Remarques à propos de la démonstration de l'unicite de P.A. Meyer, Séminaire Brelot-Choquet-Deny (Théorie de Potential) 6 (1962), No. 8, 13 pp.

10. D. Dacunha-Castelle and J. L. Krivine, Sous-Espaces de $L^{1}$, Israel J. Math. 26 (1977), 320-350.

11. L. E. Dor, On sequences spanning a complex $l^{1}$ space, Proc. Amer. Math. Soc. 47 (1975), 515-516. 
12. N. Dunford and J. T. Schwartz, Linear operators. I, General theory, Pure and Appl. Math., Vol. 7, Interscience, New York, 1958.

13. A. Dvoretsky, Some results on convex bodies and Banach spaces, Proc. Internat. Sympos. on Linear Spaces, Jerusalem, Academic Press, Jerusalem, 1961, pp. 123-160.

14. E. E. Ellentuck, A new proof that analytic sets are Ramsey, J. Symbolic Logic 39 (1974), 163-165.

15. P. Enflo, Banach spaces which can be given an equivalent uniformly convex norm, Israel J. Math. 13 (1972), 281-288.

16. H. Fakhoury, Sur les espaces de Banach ne contenant pas $l^{1}(N)$ (to appear).

17. J. Farahat, Espaces de Banach contenant $l_{1}$ d'après $H$. P. Rosenthal, Seminaire MaureySchwartz, Ecole Polytechnique, 1973-1974.

18. T. Figiel and W. B. Johnson, $A$ uniformly convex Banach space which contains no $l_{p}$, Compositio Math. 29 (1974), 179-190.

19. F. Galvin and K. Prikry, Borel sets and Ramsey's theorem, J. Symbolic Logic 38 (1973), 193-198.

20. J. Hagler, Some more Banach spaces which contain $l^{1}$, Studia Math. 46 (1973), 35-42.

21. Nonseparable "James tree" analogues of the continuous functions on the Cantor set, Studia Math. 61 (1977), 41-53.

22. appear).

23. J. Hagler and W. B. Johnson, On Banach spaces whose dual balls are not weak* sequentially compact, Israel J. Math. (to appear).

24. J. Hagler and E. Odell, $A$ Banach space not containing $l_{1}$ whose dual ball is not weak*sequentially compact, Illinois J. Math. (to appear).

25. R. Haydon, Some more characterizations of Banach spaces containing $l_{1}$, Math. Proc. Cambridge Philos. Soc. 80 (1976), 269-276.

26. On Banach spaces which contain $l^{1}(\tau)$ and types of measures on compact spaces, Israel J. Math. (to appear).

27. F. Hausdorff, Set theory, Chelsea, New York, 1962.

28. R. Huff and P. D. Morris, Dual spaces with the Krein-Milman property have the RadonNikodym property, Proc. Amer. Math. Soc. 49 (1975), 104-108.

29. R. C. James, Bases and reflexivity of Banach spaces, Ann. of Math. 52 (1950), 518-527.

30. __ Uniformly nonsquare Banach spaces, Ann. of Math. 80 (1964), 542-550.

31. _ A separable somewhat reflexive Banach space with nonseparable dual, Bull. Amer. Math. Soc. 80 (1974), 738-743.

32. W. B. Johnson and H. P. Rosenthal, On $w^{*}$-basic sequences and their applications to the study of Banach spaces, Studia Math. 43 (1972), 77-92.

33. W. B. Johnson and M. Zippin, Subspaces and quotient spaces of $\left(\Sigma G_{n}\right)_{p}$ and $\left(\Sigma G_{n}\right)_{c_{0}}$, Israel J. Math. 17 (1974), 50-55.

34. M. I. Kadec and A. PeKczyński, Bases, lacunary sequences and complemented subspaces in the spaces $L_{p}$, Studia Math. 21 (1962), 161-176.

35. J. L. Krivine, Sous-espaces de dimension finie des espaces de Banach reticules, Ann. of Math.

36. K. Kuratowski, Topology.I, Academic Press, New York, 1966.

37. J. Lindenstrauss and C. Stegall, Examples of separable spaces which do not contain $l_{1}$ and whose duals are nonseparable, Studia Math. 54 (1975), 81-105.

38. J. Lindenstrauss and L. Tzafriri, Classical Banach spaces.I; Sequence spaces, SpringerVerlag, Berlin-Heidelberg-New York, 1977.

39. B. Maurey and H. P. Rosenthal, Normalized weakly null sequences with no unconditional subsequence, Studia Math. 61 (1977), 77-98.

40. C. St. J. A. Nash-Williams, On well quasi-ordering transfinite sequences, Proc. Cambridge Philos. Soc. 61 (1965), 33-39.

41. A. Nissenzweig, $\omega^{*}$ sequential convergence, Israel J. Math. 22 (1975), 266-272.

42. E. Odell and H. P. Rosenthal, A double-dual characterization of separable Banach spaces containing $l^{1}$, Israel J. Math. 20 (1975), 375-384.

43. A. Pekczyński, On Banach spaces containing $L_{1}(\mu)$, Studia Math. 30 (1968), 231-246.

44. __ On C (S) subspaces of separable Banach spaces, Studia Math. 31 (1968), 513-522.

45. R. Phelps, Lectures on Choquet's theorem, D. van Nostrand Co., Princeton, New Jersey, 1966. 
46. G. Pisier, Martingales with values in uniformly convex spaces, Israel J. Math. 20 (1975), 326-350.

47. J. D. Pryce, A device of R. J. Whitley's applied to pointwise compactness in spaces of continuous functions, Proc. London Math. Soc. 23 (1971), 532-546.

48. H. P. Rosenthal, On relatively disjoint families of measures, with some applications to Banach space theory, Studia Math. 37 (1970), 13-36 Correction-ibid (1971), 311-313.

49. 124 (1970), 205-248.

50. _ On factors of $C[0,1]$ with nonseparable dual, Israel J. Math. 13 (1972), 361-378. Correction, ibid 21 (1975), 93-94.

51. , A characterization of Banach spaces containing $l^{1}$, Proc. Nat. Acad. Sci. U.S.A. 71 (1974), 2411-2413.

52. __ Point-wise compact subsets of the first Baire class, with some applications to Banach space theory, Aarhus Universitet various Publications Ser. No. 24, Denmark, 1974, pp. 176-187.

53. _ The Banach spaces $C(K)$ and $L^{p}(\mu)$, Bull. Amer. Math. Soc. 81 (1975), 763-781.

54. __ Point-wise compact subsets of the first Baire class, Amer. J. Math. 99 (1977), $362-378$.

55. _ On a theorem of J. L. Krivine concerning block finite-representability of $I^{P}$ in general Banach spaces, J. Functional Analysis 28 (1978).

56. __ Banach spaces generated by convergent sequences of sets (to appear).

57. A. Sobczyk, Projection of the space $m$ onto its subspace $c_{0}$, Bull. Amer. Math. Soc. 47 (1941), 938-947.

58. C. P. Stegall, The Radon-Nikodym property in conjugate Banach spaces, Trans. Amer. Math. Soc. 206 (1975), 213-223.

59. J. Stern, A Ramsey theorem for trees, with an application to Banach spaces, Israel J. Math. (to appear).

60. W. Szlenk, The non-existence of a separable reflexive Banach space universal for all separable reflexive Banach spaces, Studia Math. 30 (1968), 53-61.

61. B. S. Tsirelson, Not every Banach space contains $l^{p}$ or $c_{0}$, Functional Anal. Appl. 8 (1974), 138-141 (translated from Russian).

62. L. Tzafriri, On Banach spaces with unconditional bases, Israel J. Math. 17 (1974), $84-93$.

63. W. A. Veech, Short proof of Sobczyk's theorem, Proc. Amer. Math. Soc. 28 (1971), 627-628.

64. M. Zippin, The separable extension problem, Israel J. Math. 26 (1977), 372-387.

Department of Mathematics, University of Illinols at Urbana-Champaign, URbana, ILLINOIS 61801 